\title{
Colloquium: Gripped by light: Optical binding
}

\author{
Kishan Dholakia* \\ School of Physics and Astronomy, University of St. Andrews, North Haugh, Fife KY16 \\ 9SS, Scotland
}

Pavel Zemánek ${ }^{\dagger}$

Institute of Scientific Instruments of the ASCR, v.v.i., Academy of Sciences of the Czech Republic, Kralovopolska 147, 61264 Brno, Czech Republic

(Published 3 June 2010)

\begin{abstract}
The light-matter interaction has been at the heart of major advances from the atomic scale right to the microscopic scale over the past four decades. Confinement by light, embodied by the area of optical trapping, has had a major influence across all of the natural sciences. However, an emergent and powerful topic within this field that has steadily merged but not gained much recognition is optical binding: the importance of exploring the optically mediated interaction between assembled objects that can cause attractive and repulsive forces and dramatically influence the way they assemble and organize themselves. This offers routes for colloidal self-assembly, crystallization, and organization of templates for biological and colloidal sciences. In this Colloquium, this emergent area is reviewed looking at the pioneering experiments in the field and the various theoretical approaches that aim to describe this behavior. The latest experimental studies in the field are reviewed and theoretical approaches are now beginning to converge to describe the binding behavior seen. Recent links between optical binding and nonlinearity are explored as well as future themes and challenges.
\end{abstract}

DOI: $10.1103 /$ RevModPhys.82.1767

PACS number(s): 42.50.Wk, 87.80.Cc, 42.25.Fx

\section{CONTENTS}

I. Introduction

II. The Pioneering Experiments of Optical Binding

A. Lateral optical binding

B. Longitudinal optical binding

III. Theoretical Models

A. Optical binding of nanoparticles

1. General model for two nanoparticles

2. Lateral binding of two identical spherical nanoparticles

3. Longitudinal binding of two spherical nanoparticles

4. Optically induced forces between two nanotubes

5. Optical binding of many nanoparticles

6. Dipole near a planar surface

B. Optical binding of micro-objects

1. Optical forces

2. Optical binding of spheres

3. Paraxial optics theory for larger spheres

4. Optical binding of infinite cylinders

5. Optical binding using the coupled dipole method

6. Optical binding near a flat surface

C. Dynamical phenomena in optical binding of micro-objects

1. Particle dynamics due to optical forces

*kd1@st-andrews.ac.uk
2. Hydrodynamic equations

1782

IV. Further Experimental Studies and Configurations 1783
1767

1769

1769

1770

1771

1771

1771

1773

1775

1776

1776

1776

1777

1777

1777

1778

1779

1779

1781

1781

1781

\section{INTRODUCTION}

The momentum of light and the exertion of optical forces have had a profound and far-reaching impact in the past four decades. These concepts have been at the core of a revolution in the interaction of light with atomic systems that has led to the topics of laser cooling (Chu, 1998; Cohen-Tannoudji, 1998; Phillips, 1998) and Bose-Einstein condensation (Ketterle, 2002). The mechanical effects of light have also manifested themselves upon systems at larger size scales, notably through the controlled motion of microscopic particles where light can readily trap and move particles with no physical contact or damage whatsoever (Neuman and Block, 2004; Dholakia and Lee, 2008; Jonáš and Zemánek, 2008). In particular, this ability to move colloidal microparticles around at will has led to some spectacular and farreaching advances in the biological sciences: when used as a force transducer the position of a trapped particle can be determined to subnanometer level accuracy and allow one to monitor motion of macromolecules that are attached to such particles using surface chemistry. Other 
major areas of science have benefited from such optical forces: microrheology of polymers, studies of the laws of thermodynamics and Brownian dynamics at the microscopic scale, and optical angular momentum transfer. Additionally the interaction of particles suspended in a flowing fluid with an optical field (commonly termed an "optical potential energy landscape") has led to interesting dynamics and the concepts of optical sorting and fractionation. In the domain of colloidal science selforganization and assembly of microscopic objects enable the study of phase-transition-like behavior and explore interaction potentials as-in addition to light-induced organization-we can tune interactions with the surface chemistry of the particles themselves and by judicious choice of solvent. Colloids have a well-defined thermodynamic temperature and can give insight into processes at the atomic scale with the added benefit of individual particle monitoring due to sophisticated techniques in video tracking software.

At the heart of optical trapping there is the interplay between what are commonly termed the gradient and scattering forces. The gradient force can be understood in a number of ways. For small objects (nanoscale) it is best understood as the interaction of an induced dipole with the field gradient of the focused electromagnetic field: the dipole positions itself at the point of the highest field to minimize its interaction energy. For large diameter particles, with the diameter exceeding significantly the light wavelength, one can invoke the refraction of light by the particle that again leads to a force upon the object pulling it to the location of the maximal field intensity. These particle behaviors apply for the case of particles of higher refractive index than their surroundings. Scattering forces naturally play a role too. These forces arise due to the light momentum change upon the scattering from an object and generally point in the direction of the light propagation. In a single beam gradient trap, termed "optical tweezers," the presence of scattering causes the equilibrium position of the particle to be displaced in the direction of the beam propagation slightly beyond the beam focus. In another highly popular trapping geometry, that of a dual beam counter propagating $(\mathrm{CP})$ trap, the scattering force is dominant in keeping the particle equilibrium position between the two incident beams.

The phenomenon of optical binding represents a rather specific manifestation of light-induced forces. In contrast to the above-discussed gradient and scattering forces, which arise directly from the incident field, the binding forces rely on the modification of the incident field in the presence of multiple simultaneously illuminated objects. These objects interact mutually through the light scattering and, consequently, organize themselves into specific static or dynamic configurations. The topic of optical binding has come to the fore within the past decade. Although it was originally considered to be a mere niche in the general field of optical trapping and manipulation, it has actually turned out to be a rich diverse area of its own featuring some fascinating physics.

In this Colloquium we describe and review the emer- gent topic of "optical binding." Even though this term is well accepted and used by the community it is perhaps not well defined nor clearly understood within the context of the broad topic of optical micromanipulation. From the historical perspective optical binding generally refers to the generation of stable spatial configurations of microparticles following light illumination where the light redistribution by a given object can influence the equilibrium position of its neighbors and vice versa. These microparticles have well-defined interparticle distances and their stable configuration is reached through the interplay of the scattered photons, the physical attributes of the particles and the surrounding medium, and the illuminating light field. Commonly colloidal objects interact over long distances through electrostatic or magnetic interactions. These types of interparticle coupling mechanisms require special particles, for example, application of surface-treatment or material properties such as superparamagnetism, to be effective and, hence, they lie outside of the scope of this article. Here we focus on the laser light-mediated coupling, optical binding, that is typically much weaker than electrostatic or magnetic interactions but that is active for a wide range of particles made of various materials. It is noteworthy to mention that optical binding leads not only to attractive forces but also to repulsive forces; therefore the term "binding" is perhaps a little misleading. For the purposes of this paper we define optical binding as the mechanism based on the light-mediated momentum exchange between particles leading to spatial configurations of particles or clusters of particles with distances between them larger than the extent of any electrostatic forces from any surface charges present.

Why has the topic of optical binding become so interesting? Generally the interparticle forces produced by light scattering have different properties compared to those of electrostatic interactions. For example, they are not conservative and have a varying dependence upon the interparticle separation. However, they are also able to create the so-called "optically bound matter" where the dynamics of optically bound particles are more complex when compared to bound atoms, which leads to numerous spectacular experimental phenomena. These phenomena are rather disparate and lead to an interesting area of complexity with optical fields and new links to topics such as nonlinear optics and photonic crystals. In contrast to the single beam optical trapping and multiple beam optical trapping, in binding no predescribed optical traps are generated through the spatial shaping of the incident beam. The stable configuration between objects can be established even in a homogeneous beam (at least along the beam propagation axis) as a result of light momentum redistribution in the incident beam by the objects together with the light scattered from one object toward the others. Therefore, self-arranged configurations of colloids with micrometer-scale interparticle distances may be realized even by plane-wave illumination of the sample.

For a long time the mechanical effects of the light scattered between particles were ignored in multipar- 
ticle optical manipulation because it was expected that for a small number of microparticles they were negligible compared to the optical forces arising from the incident beams. However, experimental observations proved that this is not the case and that this redistributed light momentum can greatly influence the equilibrium positions of other adjacent colloidal objects. Therefore, a more complete description of this interaction among many particles through light would lead not only to enhance understanding of the theory of optical forces but also of the cumulative role of other relevant interactions at this scale (hydrodynamic coupling, electrostatic, radiometric, thermally driven stochastics, etc.). This could provide a powerful and unexplored route toward the colloidal self-assembly and creation of microscopic and mesoscopic templates. This is an exciting and potentially powerful route for a myriad of studies; examples include the creation of templates that may then subsequently seed nucleation of larger crystals. From a colloid scientists' viewpoint this facilitates studies of phase transitions and may even create structures that exhibit bandgap behavior (e.g., photonic crystals). As we shall see, the particle-particle interactions mediated by light are pushing our understanding of nonlinearity and complexity in colloidal science. Binding leads to feedback which in turn means we can see bistability, multistability, and nonlinear responses. We know from the early work of Ashkin and Smith how colloidal particles can act as nonlinear media (Ashkin et al., 1982; Smith et al., 1982): with optical forces pulling particles into bright field lines one can see how a modulation of the refractive index might occur in such systems. Optical binding is not only restricted to colloidal objects but it is also applicable to living cells (Buican et al., 1987; Metzger et al., 2005). Exploitation of optically assisted organization of cells is still at its infancy; however, the topic holds promise as the control of initial cell patterns dictates subsequent cell growth for tissue engineering, cell signaling studies, and other studies in synthetic biology (Akselrod et al., 2006).

In the remainder of this review we discuss optical binding and provide an overview of the key experiments and theoretical approaches in this area. While optical trapping has been in existence for over 40 years, one must note that the field of optical binding is far from being mature or well developed: we elucidate upon the present results obtained and challenges this field faces as well as indicate some future approaches that will lead to new discoveries. We begin with a review of the pioneering experimental studies that have been performed and comment on the theoretical approaches that are pertinent to the experiments. We then discuss more elaborate experiments that give further insights and finally summarize our findings with an emphasis on where further work needs to be carried out.

\section{THE PIONEERING EXPERIMENTS OF OPTICAL BINDING}

The history of optical binding of microparticles is a good example of an experimentally driven phenomenon: the pioneering observations were reported first and the related substantiating theoretical explanations came several years later. At the time of these experiments only theoretical predictions for optical binding of molecules were available (Thirunamachandran, 1980). Based on the experiments optical binding has been subdivided into two areas: so-called transverse (lateral) binding and longitudinal binding. For the transverse binding, the incident light field propagates orthogonally to the direction of the binding process. For longitudinal optical binding, the light field propagates in the direction of organization of the particle chains. This is somewhat arbitrary and based mainly on the experimental configurations used as a fully comprehensive theoretical basis of optical binding would naturally encompass both of these binding topics into one.

In this section we review the major experimental achievements together with their intuitive explanations. A detailed discussion of the available theories will be presented in Sec. II.A.

\section{A. Lateral optical binding}

The first key studies of optical binding were performed by Burns, Fournier, and Golovchenko (1989, 1990) soon after the discovery of optical tweezers (Ashkin et al., 1986). They studied the self-arrangement of dilute colloidal suspensions of polystyrene spheres placed on a substrate and illuminated by an interference field composed of up to five beams. The created optical lattice confined microspheres in the maxima of light interference patterns and pushed them, due to the radiation pressure, against the top surface of the sample cell. This configuration produced well-ordered colloidal crystals with lattice properties defined by the optical lattice. They also illuminated the colloidal sample with a single large diameter beam and observed a close-packed crystal in the center of the beam; this was the key step in observing the "strange" behavior of adjacent spheres approaching the crystal nucleus from the beam periphery. They realized this was caused by the light scattering and designed a new experiment where a single beam was shaped into a narrow ribbon - a line of light (see Fig. 1). In this configuration, the particles inside the beam were pushed by the radiation pressure in the light propagation direction to the top surface of the sample cell. Laterally they were localized in the direction perpendicular to the light line in the high-intensity region of the beam via the "gradient optical forces" that we described earlier. If two spheres of diameter $1.43 \mu \mathrm{m}$ were placed together into the beam (vacuum wavelength of $515.5 \mathrm{~nm}$ ), they formed a bound structure with preferred interparticle distances between 4 and 11 laser light wavelengths. Due to the thermal activation (Brownian motion), the interparticle distance changed, however, the analyses of the experiment revealed the existence of well-defined minima in the interaction energy (bound states) separated approximately by one laser wavelength (see Fig. 2). Based on the simplified theory of two induced dipole 


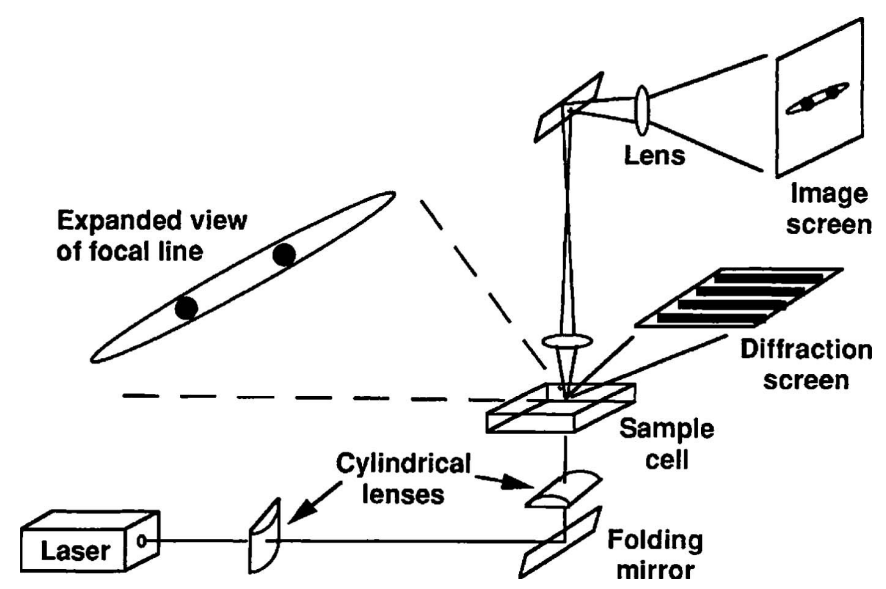

FIG. 1. Experimental setup of the first experiment with lateral optical binding. Ar laser of $10 \mathrm{~W}$ power and wavelength of $541.5 \mathrm{~nm}$ transformed into line focus was used. From Burns et al., 1989.

oscillators separated by $R$ they presented the interaction energy $W$ in the form

$$
W \approx-\frac{1}{2} \alpha^{2} k^{2} \frac{\cos (k R)}{R}|E|^{2},
$$

where $\alpha$ is the polarizability of the oscillator, $k=2 \pi / \lambda$ is the wave number in the medium surrounding the oscillator, and $E$ is the amplitude of the electric component of the light. The presented simplified formula is valid if the polarization of the incident light is perpendicular to the direction connecting the oscillators. Even though the used particles were quite large to be described by the induced dipoles, it was proven that such an approximation gave good predictions of the principal behavior of the studied system. We explore this theory in detail later. Surprisingly this pioneering experiment was largely neglected for a long time before interest in this topic was renewed through new experiments and initiated new theoretical effort. To date there remains the unanswered question of the role of the surface proximity in the presented optical binding studies as it is known that even a

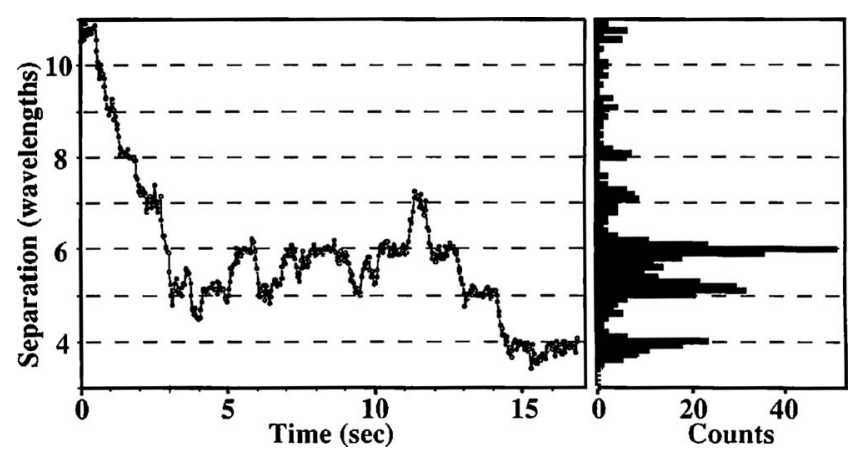

FIG. 2. Lateral optical binding in a line focus. The separation of two polystyrene spheres shown of diameters $1.43 \mu \mathrm{m}$ placed in water and illuminated with laser wavelength $541.5 \mathrm{~nm}$. Left: Time development of interparticle separation distance. Right: Histogram of the particle separation distances. From Burns et al., 1989. (a)
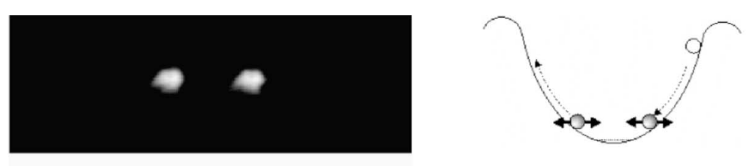

(b)
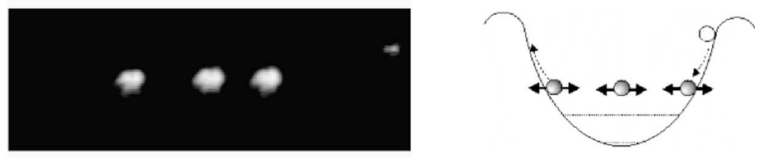

(c)
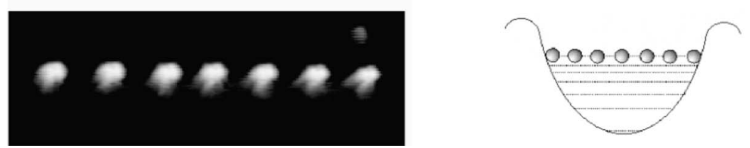

FIG. 3. Longitudinal optical binding in counterpropagating beam geometry. Experimental data for the arrays of (a) two, (b) three, and (c) seven spheres (each $3 \mu \mathrm{m}$ in size). The diagrams on the right elucidate how the particles fill up the approximately harmonic potential well created by the two counterpropagating beams. From Tatarkova et al., 2002.

weak light wave back reflected from the water-glass interface can significantly influence the behavior of single particle near the surface (Jonáš et al., 2001; Jákl et al., 2003; Fujiwara et al., 2004).

\section{B. Longitudinal optical binding}

Investigation of optical binding received a major impetus in 2002-2003 when two groups (Tatarkova et al., 2002; Singer et al., 2003) independently performed studies using the so-called counterpropagating dual beam trap which was similar to the first optical trap realized by Ashkin in 1970 (Ashkin, 1970). This trap is based on two weakly focused counterpropagating light beams with beam foci displaced in such a way the beams push the particles with the scattering force (radiation pressure) longitudinally to a location between the beam foci where both forces compensate each other. Laterally the gradient force is sufficient to confine the particles to the laser beam propagation axis. Both groups used wider diameter beams where the longitudinal changes of the optical intensity were much smaller compared to single beam trapping in optical tweezers. Perhaps surprisingly they observed that when multiple particles entered the central trap region, the particles did not coagulate, as might be expected, but rather assumed equilibrium positions with spacings that were several times the particle diameters (see Fig. 3). This effect was not caused by the interference between two counterpropagating incident light fields because they used mutually incoherent beams. This self-arrangement-termed "longitudinal" optical binding - was observed along the propagation axis (called one-dimensional binding) but far from any surface and so in three dimensions.

Electrostatic interactions could potentially play a role in such particle array formation. The interaction between charged particles can be described by the Derjaguin-Landau-Verwey-Overbeek theory (Derjaguin and Landau, 1941; Verwey and Overbeek, 1948). Ac- 
cording to this theory, the range of the electrostatic interaction is limited due to the particle charge screening in the presence of ions in the immersion medium. For the experimental parameters of Tatarkova et al. the characteristic screening length is short $(\ll 100 \mathrm{~nm})$ and the electrostatic interactions are restricted to a length scale of much less than a micron, an order of magnitude lower than the interparticle spacing observed in the bound particle arrays. Reduction of the screening length by dispersing the spheres in $1 \mathrm{M} \mathrm{NaCl}$ led to no discernible change in the observed interparticle spacing. This validated their premise that the arrays were created solely by optical forces.

In their experiment Tatarkova et al. used a geometry close to that of the original Ashkin study in 1970 (Ashkin, 1970). This comprised of two free space counterpropagating incoherent Gaussian beams. Experimentally they tracked the equilibrium positions of the spheres for bound arrays containing two to seven particles. They observed that the particles in the middle of an optically bound cluster are closer to each other when compared to the outermost particles. In order to describe the stability of the bound particle arrays, they used a quantitative model of diffusion activated escape from a one-dimensional potential well, assuming the overdamped equation of particle motion due to the viscous drag acting on the particles in the immersion liquid medium. The residence time for the spheres was studied as a function of the number of bound spheres. Furthermore, their experimental data provided quantitative information on the exact form of the trapping potential and allowed them to calculate the axial corner frequency $f_{c}=k /(2 \pi b)$, with $b$ denoting the viscous damping term. By fitting the experimental data to a parabolic potential model with the force proportional to the displacement, they were able to determine the corner frequency of the order from 100 to $1000 \mathrm{~Hz}$. Besides examining the static case they also provided the first observation of the dynamic behavior of the optically bound particle structures-breathing modes and oscillations of the center of mass. The lowest center-of-mass oscillation mode of the longitudinally bound chain was observed, which corresponds to synchronous motion of all particles in one direction.

Later they presented a detailed theoretical treatment of the longitudinal optical binding based on the paraxial wave optics (see Sec. III.B.3) which provided an intuitive but illustrative explanation of the binding based on the experimental observation of field distribution between bound particles (McGloin et al., 2004; Metzger, Wright, and Dholakia, 2006). Within their approach, small spherical particles are treated as small lenses of focal length $f=a /\left(n_{p}-n_{m}\right)$, where $a$ is the particle radius and $n_{p}$ and $n_{m}$ denote the refractive index of the particle and the immersion medium, respectively. Each of these microlenses focuses the incident beams and creates an optical trap for its neighbors that in turn create similar traps for the first particle. Therefore there exists a built-in feedback mechanism stabilizing the positions of the microparticles.
Independently of the Tatarkova et al. studies, a similar experiment was performed by Singer et al. (2003). In this case they used counterpropagating beams coming from single-mode fibers [the so-called dual beam fiber trap (Constable and Kim, 1993)] that were potentially more robust than two free space light fields. They investigated polystyrene spheres of diameters from 0.79 to $10 \mu \mathrm{m}$ and observed that spheres of diameters $0.79,1.44$, and $2 \mu \mathrm{m}$ arranged themselves into a chain with characteristic interparticle spacings that increased with increasing particle sizes. On the other hand, the distances between the particles decreased with increasing number of objects in the chain. Changes of the laser power in one beam did not affect the interparticle distances and changes of the immersion medium refractive index influenced them only slightly. An increase of the separation between the fiber ends also slightly increased the interparticle spacing. They observed that spheres larger than $2 \mu \mathrm{m}$ arranged themselves into a close-packed chain where the objects touched each other.

They developed a simplified theoretical description that supported their observations; their model assumed that the particle chain scatters light similarly to a diffraction grating illuminated by a plane wave. With the help of experimental observations they matched the transverse periodicity of the distributed light with the interparticle periodicity and obtained good coincidence between the observations and theory. However, some deviations from their theory were expected because it has been shown that the interparticle distances are not the same and the lateral beam has a Gaussian profile in fact rather than that of a plane wave.

\section{THEORETICAL MODELS}

\section{A. Optical binding of nanoparticles}

We start this section dedicated to the theoretical description of optical binding by studying the interaction between two radiating induced dipoles representing either symmetric (nanospheres) or asymmetric (nanotubes and molecules) nanoparticles with no permanent electric dipole. The radiation from the dipoles is a consequence of charge oscillation induced by the incident electromagnetic field. Even though a detailed quantitative experiment dealing with nanoparticle binding has yet to be reported, we have seen in Sec. II.B that such a theoretical approximation can give analytical results with a reasonable first-order explanation of the problem. Consequent analyses with more sophisticated theories can then follow and refine these results.

\section{General model for two nanoparticles}

In this section we use the classical model of induced oscillating dipoles to express the forces acting upon them. We denote the induced dipole moments $\mathbf{p}\left(\mathbf{r}_{A}\right)$ and $\mathbf{p}\left(\mathbf{r}_{B}\right)$ and place them at $\mathbf{r}_{A}$ and $\mathbf{r}_{B}$, respectively. We further assume that they are induced by the total electric field at the respective dipole locations 
$\mathbf{E}\left(\mathbf{r}_{A}, t\right)=\mathbf{E}\left(\mathbf{r}_{A}\right) \exp (-i \omega t)$ and $\mathbf{E}\left(\mathbf{r}_{B}, t\right)$ of a similar form. If we omit the time dependence, the dipole components can be expressed using the Einstein summation convention (the sign $\Sigma$ is skipped when the same index appears twice in the summation formula),

$$
p_{i}\left(\mathbf{r}_{A}\right)=\alpha_{i j}^{A} E_{j}\left(\mathbf{r}_{A}\right), \quad p_{i}\left(\mathbf{r}_{B}\right)=\alpha_{i j}^{B} E_{j}\left(\mathbf{r}_{B}\right),
$$

where $\alpha^{A}$ and $\alpha^{B}$ are the polarizability tensors of particles $A$ and $B$, respectively. Within the linear physics formalisms these dipoles oscillate at the same frequency as the incident field and, consequently, emit radiation through which they interact with the other dipole. The total electric field at the position of the dipole is given by the sum of the incident field $\mathbf{E}^{I}(\mathbf{r})$ and the field emitted by the other dipole,

$$
\begin{aligned}
& E_{i}\left(\mathbf{r}_{A}\right)=E_{i}^{I}\left(\mathbf{r}_{A}\right)+G_{i j}\left(\mathbf{r}_{A}, \mathbf{r}_{B}\right) \alpha_{j k}^{B} E_{k}\left(\mathbf{r}_{B}\right), \\
& E_{i}\left(\mathbf{r}_{B}\right)=E_{i}^{I}\left(\mathbf{r}_{B}\right)+G_{i j}\left(\mathbf{r}_{B}, \mathbf{r}_{A}\right) \alpha_{j k}^{A} E_{k}\left(\mathbf{r}_{A}\right),
\end{aligned}
$$

where we used the field propagator between two dipoles $\overline{\mathbf{G}}$ (also called the dyadic Green's function) in the form

$$
\begin{aligned}
G_{j k}= & \frac{\exp (i k R)}{4 \pi \varepsilon_{0} \varepsilon_{m} R^{3}}\left[\left(3-3 i k R-k^{2} R^{2}\right) \frac{R_{j} R_{k}}{R^{2}}\right. \\
& \left.+\left(k^{2} R^{2}+i k R-1\right) \delta_{j k}\right] .
\end{aligned}
$$

Here $R$ is the length given by $\mathbf{R}=\mathbf{r}_{B}-\mathbf{r}_{A}, k=2 \pi / \lambda$ is the wave number of the light in the medium, $\varepsilon_{0}$ is the permittivity of vacuum, $\varepsilon_{m}$ is the relative permittivity of the medium, and $\delta_{j k}$ denotes the Kronecker delta. Equation (4) contains three terms proportional to $(k R)^{-1},(k R)^{-2}$, and $(k R)^{-3}$. Each of them dominates for different distances between nanoparticles (Novotny and Hecht, 2006). The far-field term $(k R)^{-1}$ dominates at $R \gg \lambda$, whereas the near-field term $(k R)^{-3}$ dominates at $R \ll \lambda$. The last term with $(k R)^{-2}$ plays the key role in the intermediate separation distances $R \simeq \lambda$. Comparison of the spatial distribution of the amplitude for an electric field emitted by the oscillating dipole is shown in Fig. 4 for the near field and far field. Whereas in the far field the nanoparticle only weakly emits along the direction of the oscillating dipole, in the near field the electrostatic components increase the electric field amplitude along the direction of the dipole moment.

The solution of Eq. (3) for particle $B$ is

$$
E_{i}^{B}=K_{i j} E_{j}^{I B}+K_{i j} G_{j k} \alpha_{k m}^{A} E_{m}^{I A},
$$

where $K_{i j}$ denotes the components of the inverse tensor $\left[\overline{\mathbf{I}}-\overline{\mathbf{G}}\left(\mathbf{r}_{B}, \mathbf{r}_{A}\right) \alpha_{A} \overline{\mathbf{G}}\left(\mathbf{r}_{A}, \mathbf{r}_{B}\right) \alpha_{B}\right]^{-1}$ and we simplified the notation $E_{i}\left(\mathbf{r}_{A}\right) \equiv E_{i}^{A}, \quad E_{i}\left(\mathbf{r}_{B}\right) \equiv E_{i}^{B}, \quad$ and $\quad G_{i j}\left(\mathbf{r}_{A}, \mathbf{r}_{B}\right)$ $=G_{i j}\left(\mathbf{r}_{B}, \mathbf{r}_{A}\right) \equiv G_{i j}$. The tensor $K_{i j}$ includes multiple scattering between both objects. However, it differs significantly from unity only for configurations where the dipoles are close to each other. For particles of diameters in the range of tens of nanometers it fulfills the condition
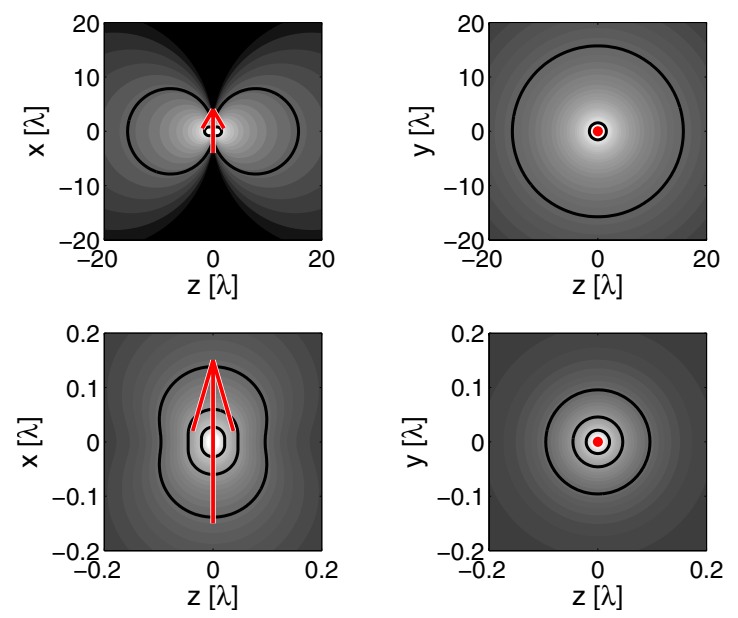

FIG. 4. (Color online) Examples of the amplitude distribution of the electric field emitted by the oscillating dipole oriented along the $x$ axis (arrow). (a) The upper row shows the far field where the emitted field amplitude decreases much faster along the $x$ axis. (b) The bottom row shows the near field where in contrast the field amplitude decreases slowly along the $x$ axis. Black contours denote places of the same field intensity; field amplitudes on neighboring contours differ by one order of magnitude.

$$
K_{i j} \cong \delta_{i j},
$$

which is coherent with the first Born approximation (Born and Wolf, 1999).

Once the total electric component of the optical field is known, the time-averaged component $F_{\xi}$ of the optical force acting on the object $B$ can be expressed as (de Groot and Suttorp, 1971; Chaumet and NietoVesperinas, 2000a)

$$
\left\langle F_{\xi}\left(\mathbf{r}_{B}\right)\right\rangle=\frac{1}{2} \operatorname{Re}\left\{\left.p_{i}^{*}\left(\mathbf{r}_{B}\right) \frac{\partial E_{i}(\mathbf{r})}{\partial r_{\xi}}\right|_{\mathbf{r}=\mathbf{r}_{B}}\right\},
$$

where $\operatorname{Re}\{X\}$ denotes the real part of $X$. To simplify the notation further, we denote the force component $F_{\xi}$ acting on the dipole $B$ as $F_{\xi}^{B}$ and partial derivatives at $\mathbf{r}$ $=\mathbf{r}_{B}$ as $\partial_{\xi}^{B}$. Therefore, Eq. (7) has the following compact form if Eq. (2) is also employed:

$$
F_{\xi}^{B}=\frac{1}{2} \operatorname{Re}\left\{\alpha_{i j}^{B *} E_{j}^{B *} \partial_{\xi}^{B} E_{i}\right\}
$$

where the indices $i, j=1,2,3$ denote the Cartesian axes $x, y, z$ and $\partial_{\xi}^{B} E_{i}$ has the form

$$
\partial_{\xi}^{B} E_{i}=\partial_{\xi}^{B} E_{i}^{I B}+\partial_{\xi}^{B}\left(G_{i k}\right) \alpha_{k m}^{A} E_{m}^{I A} .
$$

If we substitute Eqs. (5) and (9) into Eq. (8), the general optical force acting on particle $B$ can be expressed as

$$
\begin{aligned}
F_{\xi}^{B}= & \frac{1}{2} \operatorname{Re}\left\{\alpha _ { i j } ^ { B * } [ E _ { j } ^ { I B * } + G _ { j k } ^ { * } \alpha _ { k m } ^ { A * } E _ { m } ^ { I A * } ] \left[\partial_{\xi}^{B} E_{i}^{I B}\right.\right. \\
& \left.\left.+\partial_{\xi}^{B}\left(G_{i q}\right) \alpha_{q r}^{A} E_{r}^{I A}\right]\right\} .
\end{aligned}
$$

The force acting on dipole $A$ can be obtained by interchanging the letters $A$ and $B$. The total optical force (10) can be further simplified if one realizes that only particles much smaller than the radiation wavelength are 


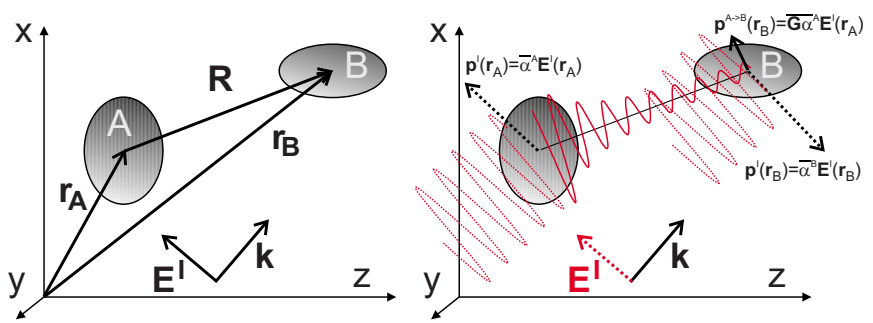

FIG. 5. (Color online) Definition of variables used for the mathematical description (left). Simplified scheme of the interaction between two particles without multiple scattering between them which is equivalent to the description given by Eq. (11). Incident field induces dipoles $\mathbf{p}^{I}\left(\mathbf{r}_{A}\right)=\bar{\alpha}^{A} \mathbf{E}^{I}\left(\mathbf{r}_{A}\right)$ or $\mathbf{p}^{I}\left(\mathbf{r}_{B}\right)$ $=\bar{\alpha}^{B} \mathbf{E}^{I}\left(\mathbf{r}_{B}\right)$ in particle $A$ or $B$, respectively. The field emitted by particle $A$ reaches particle $B$ and induces there the dipole component $\mathbf{p}^{A \rightarrow B}\left(\mathbf{r}_{A}\right)=\overline{\mathbf{G}} \bar{\alpha}^{A} \mathbf{E}^{I}\left(\mathbf{r}_{A}\right)$ which interacts with the incident field $\mathbf{E}^{I}\left(\mathbf{r}_{B}\right)$. The second parallel path corresponds to the interaction of the emitted field by the dipole $A$ with the dipole induced in particle $B$ by the incident field $\mathbf{E}^{I}\left(\mathbf{r}_{B}\right)$.

considered here. Their polarizabilities are generally very small and therefore terms with product of three and more $\alpha_{i j}$ can be omitted,

$$
\begin{aligned}
F_{\xi}^{B}= & \frac{1}{2} \operatorname{Re}\left\{\alpha_{i j}^{B_{*}^{*}} E_{j}^{I B^{*}} \partial_{\xi}^{B} E_{i}^{I B}+\alpha_{i j}^{B *} G_{j k}^{*} \alpha_{k m}^{A *} E_{m}^{I A *} \partial_{\xi}^{B} E_{i}^{I B}\right. \\
& \left.+\alpha_{i j}^{B *} E_{j}^{I B *} \partial_{\xi}^{B}\left(G_{i q}\right) \alpha_{q r}^{A} E_{r}^{I A}\right\} .
\end{aligned}
$$

The first term is the classical formula for the optical force acting upon an isolated dipole $B$ placed in an incident field $\mathbf{E}^{I B}$ without the presence of dipole $A$,

$$
{ }^{\mathrm{OF}} F_{\xi}^{B}=\frac{1}{2} \operatorname{Re}\left\{\alpha_{i j}^{*} E_{j}^{I B *} \partial_{\xi}^{B} E_{i}^{I B}\right\} .
$$

This force is determined solely by the spatial distribution of the incident field and it is usually further divided into gradient force and scattering force components (Harada and Asakura, 1996; Chaumet and NietoVesperinas, 2000a; Novotny and Hecht, 2006). The second force term in Eq. (11) describes the interaction between the incident field $E^{I}\left(\mathbf{r}_{B}\right)$ and the part of the dipole $B$ induced by the field scattered from the dipole $A$ located at $\mathbf{r}_{A}$. The third term corresponds to when the dipole is induced by the incident field at $\mathbf{r}_{B}$ but interacts with the field scattered from dipole $A$ placed at $\mathbf{r}_{A}$. The second and third terms (all depending on $\overline{\mathbf{G}}$ ) describe the coupling force acting between the nanoobjects which is mediated by the light scattering-the so-called optical binding force. Adjective binding, however, does not mean that this force is purely attractive. For optically bound particles, there exist equilibrium configurations characterized by a well-defined separation distance. If the separation of the particles becomes larger than the equilibrium one, the binding force is attractive and pulls the particles together. If, on the other hand, the separation decreases with respect to the equilibrium one, the binding force is repulsive and pushes the particles apart (see Fig. 5).

\section{Lateral binding of two identical spherical nanoparticles}

Now we simplify the above-presented equations to provide a clearer picture of the behavior of two optically bound spherical nanoparticles illuminated by an incident wave $\mathbf{E}^{I}$ propagating perpendicularly to the interparticle displacement vector $\mathbf{R}$. This geometry is similar to the pioneering experiments of Burns et al. (1989) and has been studied theoretically in detail by Depasse and Vigoureux (1994), by Nieto-Vesperinas et al. (2004), and later by Romero et al. (2008) who used a quantum electrodynamical approach.

We assume that the objects are identical isotropic spheres, i.e., $\alpha_{i j}^{A} \equiv \alpha_{i j}^{B}=\alpha \delta_{i j}$. The polarizibility $\alpha$ is in general complex and usually expressed in the form of effective polarizability also including the radiation reaction term (Draine, 1988; Novotny and Hecht, 2006)

$$
\alpha=\frac{\alpha_{0}}{1-i k^{3} \alpha_{0} /\left(6 \pi \varepsilon_{0} \varepsilon_{m}\right)} \equiv \alpha^{\prime}+i \alpha^{\prime \prime}
$$

$$
\alpha_{0}=4 \pi \varepsilon_{0} \varepsilon_{m} a^{3} \frac{\varepsilon_{p}-\varepsilon_{m}}{\varepsilon_{p}+2 \varepsilon_{m}}
$$

where $\varepsilon_{p}$ is the relative permittivity of the particle and $\alpha^{\prime}$ and $\alpha^{\prime \prime}$ are the real and imaginary parts of the polarizability $\alpha$, respectively.

Without loss of generality we assume $\mathbf{R}$ $=[0, R \sin \varphi, R \cos \varphi]$ and a linearly polarized incident plane wave propagating along $x$ axis with wave vector $\mathbf{k}=[k, 0,0]$ and having electric field components $\mathbf{E}^{I B}$ $=\mathbf{E}^{I A}=\left[0,0, E_{0} \exp (i k x)\right]$. Their substitution into Eq. (11) and using Eq. (4) give force components within the first Born approximation. Based on the symmetry of the problem it is advantageous to use the radial force component $F_{r}^{B}$ (along $\mathbf{R}$ ) and azimuthal force component $F_{\varphi}^{B}$ (perpendicular to $\mathbf{R}$ and following increase of $\varphi$ ),

$$
\begin{aligned}
& F_{r}^{B}=F_{y}^{B} \sin \varphi+F_{z}^{B} \cos \varphi, \\
& F_{\varphi}^{B}=F_{y}^{B} \cos \varphi-F_{z}^{B} \sin \varphi .
\end{aligned}
$$

They provide the following simplified form of the forces:

$$
\begin{aligned}
F_{r}^{B}= & \frac{|\alpha|^{2}\left|E_{0}\right|^{2}}{8 \pi \varepsilon_{0} \varepsilon_{m} R^{4}}\left\{\left[2 k^{2} R^{2}\left(2 \cos ^{2} \varphi-1\right)\right.\right. \\
& \left.+3\left(1-3 \cos ^{2} \varphi\right)\right] \cos (k R) \\
& +\left[k^{3} R^{3}\left(\cos ^{2} \varphi-1\right)\right. \\
& \left.\left.+3 k R\left(1-3 \cos ^{2} \varphi\right)\right] \sin (k R)\right\}, \\
F_{\varphi}^{B}= & \frac{|\alpha|^{2}\left|E_{0}\right|^{2} \sin (2 \varphi)}{8 \pi \varepsilon_{0} \varepsilon_{m} R^{4}}\left[\left(k^{2} R^{2}-3\right) \cos (k R)\right. \\
& -3 k R \sin (k R)],
\end{aligned}
$$




$$
\begin{aligned}
F_{x}^{B}= & \frac{k}{2} \alpha^{\prime \prime}\left|E_{0}\right|^{2}-\operatorname{Re}\left\{\frac{\left(\alpha^{*}\right)^{2}\left|E_{0}\right|^{2} k \exp (-i k R)}{8 \pi \varepsilon_{0} \varepsilon_{m} R^{3}}\right. \\
& \left.\times\left[3 i-3 k R-i k^{2} R^{2}\right] \sin ^{2} \varphi-2 i+2 k R\right\} .
\end{aligned}
$$

Along the $x$ axis the first term describes the scattering force $k \alpha^{\prime \prime}\left|E_{0}\right|^{2} / 2$ coming from the incident plane wave and the second term corresponds to the scattering force component caused by the interference of the incident wave with the scattered wave from the other particle. In order to better understand the interaction between particles in lateral plane we focus on the near- and far-field regimes.

In the near-field region $k R \ll 1$ we obtain [assuming $\cos (k R) \simeq 1$ and $\sin (k R) \simeq k R \ll 1]$

$$
\begin{aligned}
& F_{r}^{B}=-\frac{3|\alpha|^{2}\left|E_{0}\right|^{2}}{8 \pi \varepsilon_{0} \varepsilon_{m} R^{4}}\left(3 \cos ^{2} \varphi-1\right), \\
& F_{\varphi}^{B}=-\frac{3|\alpha|^{2}\left|E_{0}\right|^{2}}{8 \pi \varepsilon_{0} \varepsilon_{m} R^{4}} \sin (2 \varphi), \\
& F_{x}^{B}=\frac{k}{2} \alpha^{\prime \prime}\left|E_{0}\right|^{2}\left[1+\alpha^{\prime} \frac{2-3 \sin ^{2} \varphi}{2 \pi \varepsilon_{0} \varepsilon_{m} R^{3}}\right] .
\end{aligned}
$$

The dominant interaction between the dipoles in the near field is due to the electrostatic field which justifies using a quasielectrostatic approximation to explain the dipoles behavior. If $\varphi=0$, both nanoparticles are placed on the $z$ axis and since the electric field vector has only a $z$ component, it induces dipoles oriented along their connecting line $(\rightarrow \rightarrow)$. Hence, the positive end of one dipole is close to the negative end of the other, they attract each other, and $F_{r}^{B}$ is negative. If $\varphi>0$ and $\varphi$ $<\pi / 2$, the force $F_{\varphi}^{B}$ is negative pushing particle $B$ toward the $z$ axis. Consequently, a torque acts on the system of both dipoles that tends to orient them so that their interconnection line is again parallel to $z$ and consequently to $\mathbf{E}^{I B}$. A certain value of $\varphi$ gives positive $F_{r}^{B}$ but together with negative $F_{\varphi}^{B}$ both forces drag the particles toward the $z$ axis. If $\varphi=\pi / 2$, the force $F_{r}^{B}>0$ and the nanoparticles tend to separate because their positive and negative parts of induced and parallel dipoles $(\uparrow \uparrow)$ repel each other. In this case the force $F_{\varphi}^{B}=0$; however, this configuration is unstable and small variations in particle position generate nonzero $F_{\varphi}^{B}$ pushing the dipole $B$ toward the $z$ axis to a new position with vectors parallel to the electric field and $\mathbf{R}$. To conclude, in the near field both nanoparticles tend to orient in such a way that their interconnecting line is parallel to the electric field vector and afterward both particles approach each other (see Fig. 6).

In the far-field region $k R \gg 1$ we obtain

$$
\begin{aligned}
F_{r}^{B}= & \frac{|\alpha|^{2}\left|E_{0}\right|^{2} k^{2}}{8 \pi \varepsilon_{0} \varepsilon_{m} R^{2}}\left[k R\left(\cos ^{2} \varphi-1\right) \sin (k R)\right. \\
& \left.+2\left(2 \cos ^{2} \varphi-1\right) \cos (k R)\right],
\end{aligned}
$$
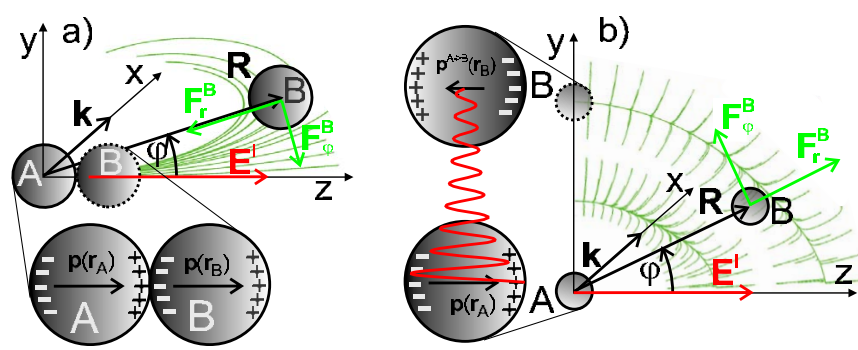

FIG. 6. (Color online) Visualization of the forces acting on particle $B$ if particle $A$ is placed at the origin of the coordinate system. (a) The near- and (b) far-field cases, respectively. Curves at the background follow the trajectories of particle $B$ toward equilibrium positions (denoted by the dotted edges).

$$
\begin{aligned}
F_{\varphi}^{B}= & \frac{|\alpha|^{2}\left|E_{0}\right|^{2} k^{2}}{8 \pi \varepsilon_{0} \varepsilon_{m} R^{2}} \sin (2 \varphi) \cos (k R), \\
F_{x}^{B}= & \frac{k}{2} \alpha^{\prime \prime}\left|E_{0}\right|^{2}+\frac{\left|E_{0}\right|^{2} k^{3} \sin ^{2} \varphi}{8 \pi \varepsilon_{0} \varepsilon_{m} R}\left[\left(\alpha^{\prime 2}-\alpha^{\prime \prime 2}\right) \sin (k R)\right. \\
& \left.+2 \alpha^{\prime} \alpha^{\prime \prime} \cos (k R)\right] .
\end{aligned}
$$

Under these conditions $F_{r}^{B} \sim-\left(1-\cos ^{2} \varphi\right) \sin (k R) / R$ is the dominant force component due to its $1 / R$ dependence on the nanoparticle separation distance. It reaches its extreme value for $\varphi=\pi / 2$ where the induced dipoles in the nanoparticles are parallel to each other and perpendicular to their connecting line $(\uparrow \uparrow)$. This dominant force term describes the force acting on the dipole $B$ that is induced by the incident plane wave and placed into the field radiated by the dipole $A$. As the distance between the dipoles increases, the induced dipole $B$ maintains its orientation and size because the amplitude and phase of the incident field do not change. However, the field radiated by dipole $A$ changes its magnitude and phase at the position of dipole $B$ and this causes the force oscillations described by $\sin (k R)$ and $\cos (k R)$. The stable configuration of these dipoles is determined by $\sin (k R)=0$ with $k R=2 M \pi$, where $M$ denotes larger natural number. Therefore, there exist several stable radial configurations of the two-dipole chain that differ by approximately the wavelength $\lambda$ of the illuminating light in the medium. The second term $\cos (k R)$ only negligibly varies this equilibrium position if $k R \gg 1$. The force $F_{\varphi}^{B}$ is much weaker compared to $F_{r}^{B}$ due to its $1 / R^{2}$ dependence, but if the particle is placed in the equilibrium position $[\sin (k R)=0], F_{\varphi}^{B}$ determines that the particles tend to orient parallel to the $y$ axis, i.e., perpendicular to the electric field vector (see Fig. 6). Equation (22) also shows that if the dipoles are parallel to the interconnecting line $(\rightarrow \rightarrow)$, the binding force is much weaker and follows as $\cos (k R) /(k R)^{2}$. This behavior is related to the fast decay of the emitted field amplitude along the direction of the induced dipole moment (see Fig. 4). 

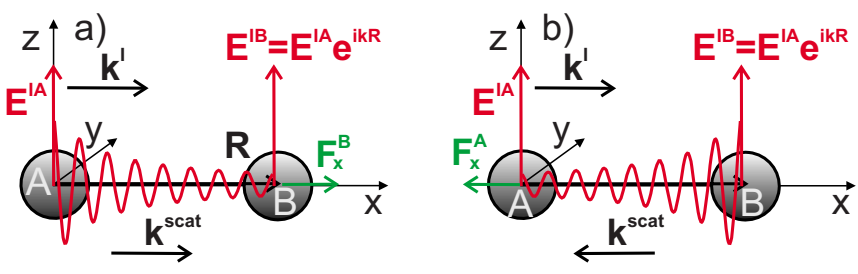

FIG. 7. (Color online) Interaction between spherical nanoparticles $A$ and $B$ in the case of longitudinal binding. Sphere $A$ radiates toward sphere $B$ in the same direction as the incident field and with the same wave vector size. Therefore the phase of the incident and scattered field is independent of the interparticle distance $\mathbf{R}$ (left) if sphere $A$ is fixed. In contrast the sphere $B$ radiates backward against the incident wave propagation, thus the phase of the incident and scattered waves depends on the interparticle distance as $2 k R$ and it results in oscillatory behavior of force $F_{x}^{A}$.

\section{Longitudinal binding of two spherical nanoparticles}

We now focus on a different configuration where the interparticle vector $\mathbf{R}=[R, 0,0]$ is parallel to the direction of the incident plane-wave propagation. Similar as above we consider the incident electric field in the form $\mathbf{E}^{I A}=\left[0,0, E_{0}^{I A} \exp [i k x]\right], \quad \mathbf{E}^{I B}=\left[0,0, E_{0}^{I B} \exp [i k(x+R)]\right]$ (see Fig. 7) and identical spherical particles satisfying $\alpha_{i j}=\delta_{i j} \alpha$. Inserting this form of the incident field into Eq. (11) we obtain $F_{z}^{B}=F_{z}^{A}=F_{y}^{B}=F_{y}^{A}=0$ and

$$
\begin{aligned}
F_{x}^{B}= & \frac{1}{2} \operatorname{Re}\left[\alpha^{*} E_{z}^{I B *} \partial_{x}^{B} E_{z}^{I B}+\alpha^{*} G_{z z}^{*} \alpha^{*} E_{z}^{I A *} \partial_{x}^{B} E_{z}^{I B}\right. \\
& \left.+\alpha^{*} E_{z}^{I B *} \partial_{x}^{B}\left(G_{z z}\right) \alpha E_{z}^{I A}\right] .
\end{aligned}
$$

The first term is the classical optical force arising from the interaction between the dipole $B$ induced and the incident field $E^{I}\left(\mathbf{r}_{B}\right)$. The second term describes the interaction between the incident field $E^{I B}$ and the part of the dipole $B$ induced by the field scattered from the dipole $A$ located at $\mathbf{r}_{A}$. The third term corresponds to when the dipole is induced by the incident field at $\mathbf{r}_{B}$ but interacts with the field scattered from dipole $A$ at $\mathbf{r}_{A}$. The force acting on dipole $A$ can be obtained by interchanging the letters $A$ and $B$ in Eq. (25).

If the incident fields are substituted into Eq. (25) and Eq. (4) is utilized together with $\partial_{x}^{B} G_{z z}=-\partial_{x}^{A} G_{z z}$, one obtains

$$
\begin{aligned}
F_{x}^{A}= & \frac{k}{2} \alpha^{\prime \prime}\left|E_{0}\right|^{2}+\frac{\left|E_{0}\right|^{2}}{8 \pi \varepsilon_{0} \varepsilon_{m} R^{4}}\left\{\left[2 \alpha^{\prime 2} k^{3} R^{3}-2 k^{2} R^{2} \alpha^{\prime} \alpha^{\prime \prime}\right.\right. \\
& \left.-2\left(2 \alpha^{\prime 2}+\alpha^{\prime \prime 2}\right) k R\right] \sin (2 k R) \\
& +\left[2 \alpha^{\prime} \alpha^{\prime \prime} k^{3} R^{3}+\left(3 \alpha^{\prime 2}+\alpha^{\prime \prime 2}\right) k^{2} R^{2}\right. \\
& \left.\left.-2 \alpha^{\prime} \alpha^{\prime \prime} k R-3\left(\alpha^{\prime 2}+\alpha^{\prime \prime 2}\right)\right] \cos (2 k R)\right\} \\
F_{x}^{B}= & \frac{k}{2} \alpha^{\prime \prime}\left|E_{0}\right|^{2}+\frac{\left|E_{0}\right|^{2}}{8 \pi \varepsilon_{0} \varepsilon_{m} R^{4}}\left[2 \alpha^{\prime} \alpha^{\prime \prime} k^{3} R^{3}\right. \\
& \left.-\left(\alpha^{\prime 2}+3 \alpha^{\prime \prime 2}\right) k^{2} R^{2}-2 \alpha^{\prime} \alpha^{\prime \prime} k R+3\left(\alpha^{\prime 2}+\alpha^{\prime \prime 2}\right)\right]
\end{aligned}
$$

We can immediately see that the force $F_{x}^{A}$ oscillates with a period corresponding to the spacing between interfer- ence maxima of two interfering counterpropagating waves. This can be explained as follows. The incident field induces a dipole at $A$ and a dipole at $B$ oscillating with phase shift $k R$ relative to dipole $A$. The dipole $B$ then radiates backward toward the dipole $A$; this backward-scattered light reaches the oscillating dipole $A$ with the total phase shift of $2 k R$. The force $F_{x}^{A}$ stems from the interaction of the dipole $A$ with the interference product of the backward-scattered field and incident field at $\mathbf{r}_{A}$ (see Fig. 7). In contrast the force $F_{x}^{B}$ pushes the dipole $B$ along the wave propagation direction without such oscillations.

In the near-field region we obtain

$$
F_{x}^{B}=-F_{x}^{A}=3\left|E_{0}\right|^{2} \frac{|\alpha|^{2}}{8 \pi \varepsilon_{0} \varepsilon_{m} R^{4}} .
$$

Thus the interaction between both dipoles is repulsive and very strong if they are close to each other. This configuration is unstable and the dipoles will tend to orient into the stable lateral configuration studied above.

In the far-field region we obtain from Eq. (26)

$$
\begin{aligned}
F_{x}^{A}= & \frac{\alpha^{\prime \prime} k\left|E_{0}\right|^{2}}{2}+\frac{k^{3}\left|E_{0}\right|^{2}}{4 \pi \varepsilon_{0} \varepsilon_{m} R}\left[\alpha^{\prime 2} \sin (2 k R)\right. \\
& \left.+\alpha^{\prime} \alpha^{\prime \prime} \cos (2 k R)\right], \\
F_{x}^{B}= & \frac{\alpha^{\prime \prime} k\left|E_{0}\right|^{2}}{2}+\frac{\alpha^{\prime} \alpha^{\prime \prime} k^{3}\left|E_{0}\right|^{2}}{4 \pi \varepsilon_{0} \varepsilon_{m} R} .
\end{aligned}
$$

It is not quite clear how the particles will move; therefore we define the binding force

$$
\begin{aligned}
F_{x}^{B A}= & F_{x}^{B}-F_{x}^{A}=-\frac{\alpha^{\prime} k^{3}\left|E_{0}\right|^{2}}{4 \pi \varepsilon_{0} \varepsilon_{m} R}\left\{\alpha^{\prime} \sin (2 k R)\right. \\
& \left.+\alpha^{\prime \prime}[\cos (2 k R)-1]\right\} .
\end{aligned}
$$

If this force is positive, the particles are repelled from each other and the interparticle distance $R$ increases. In contrast if the force is negative, the particles attract and approach each other. Therefore stable positions occur at $\sin (2 k R)=0$ with $2 k R=2 M \pi$, where $M$ is larger natural number. This shows that in the far field both dipoles in the longitudinal binding geometry find several stable configurations with respect to each other; they are separated $\lambda / 2$ which is half the distance compared to the lateral binding configurations. However, such a stable chain of both dipoles moves along the $x$ axis due to the scattering force $\alpha^{\prime \prime} k\left|E_{0}\right|^{2} / 2$ resulting from the incident beam. More general treatment would show that longitudinal orientation is the most stable in the far field.

Therefore two counterpropagating beams of the same intensity are used experimentally to compensate the radiation pressure and stop this motion of the center of mass of the chain. Both beams should be incoherent so that they do not interfere and no unwanted intensity modulation (standing wave) is created. In the presence of the second counterpropagating beam of the same intensity and wavelength, additional force terms $F_{x}^{A-}$ and $F_{x}^{B-}$ have to be added to the equations derived above 
satisfying $F_{x}^{A-}=-F_{x}^{B}$ and $F_{x}^{B-}=-F_{x}^{A}$. One can easily discover that in this case the final force acting upon particle $B, F_{x}^{2 B}$ and the binding force $F_{x}^{2 B A}$ between particles satisfy the following equations in the far-field regime (Karásek and Zemánek, 2007):

$$
F_{x}^{2 B}=-F_{x}^{2 A}=F_{x}^{B A}, \quad F_{x}^{2 B A}=2 F_{x}^{B A} .
$$

\section{Optically induced forces between two nanotubes}

The above procedures can become rather complex if we assume that the particle is inhomogeneous and anisotropic and $\alpha$ can be considered as a tensor. However, a different approach based on the quantum electrodynamic formalism can be adopted for cylindrical particles. This topic has been studied by Bradshaw and Andrews (2005b) (Andrews and Bradshaw, 2005; Bradshaw and Andrews, 2005a, 2006; Andrews et al., 2006). They considered a pair of cylindrical particles elongated along one axis and determined the light-induced system energy shift $\Delta E^{\text {ind }}$ from the fourth-order perturbation theory if the particles were illuminated by a plane wave of electric field intensity $E_{0}$. The simplified expression can be derived if the long axes of both cylinders are parallel along the $x$ axis and the particles are displaced by $\mathbf{R}$ along the $z$ axis,

$$
\begin{aligned}
\Delta E^{\text {ind }}= & \frac{E_{0}^{2}}{4 \pi \varepsilon_{0}}\left[\left(a_{\perp}^{2} \sin ^{2} \phi \sin ^{2} \theta+\alpha_{\|}^{2} \cos ^{2} \phi\right)\right. \\
& \times\left(\frac{\cos (k R)}{R^{3}}+\frac{k \sin (k R)}{R^{2}}-\frac{k^{2} \cos (k R)}{R}\right) \\
& \left.-\alpha_{\perp}^{2} \sin ^{2} \phi \cos ^{2} \theta\left(\frac{\cos (k R)}{R^{3}}+\frac{k \sin (k R)}{R^{2}}\right)\right] \\
& \times \cos (\mathbf{k} \cdot \mathbf{R}),
\end{aligned}
$$

where $\alpha_{\|}$and $\alpha_{\perp}$ denote object polarizability in a direction along the nanotube long axis and perpendicular to it, respectively. $\phi$ and $\theta$ correspond to the angle of the incident electric intensity vector with respect to the $z$ axis and with respect to the $x$ axis in the $x-y$ plane. The light-induced force is then given by

$$
F^{\text {ind }}=-\frac{\partial \Delta E^{\text {ind }}}{\partial \mathbf{R}}
$$

and describes the internal force acting on the objects. This force is related to the forces previously presented as $F^{\text {ind }}=\left(F^{B}-F^{A}\right) / 2$. The approach of Bradshaw and Andrews is very general: they presented detailed formulas for a pair of nanotubes oriented parallel to each other and for a tumbling pair of nanotubes (Bradshaw and Andrews, 2005b, 2006).

\section{Optical binding of many nanoparticles}

The method in Sec. III.A can be extended to many nanoparticles by extending the system of Eqs. (3) to $N$ equations for $N$ considered objects. A numerical solution of such a system of coupled equations also forms the basis of an efficient method called coupled dipoles
(Purcell and Pennypacker, 1973; Draine, 1988), which is applicable to larger objects and will be discussed in Sec. III.B.5.

Guillon (2006) considered a linear chain of dipoles and focused on the field enhancement in such an arrangement. He considered lateral binding configuration in two counterpropagating waves and therefore the expected periodicity between neighboring particles was one wavelength. However, neglecting scattering forces he showed that this is valid only for the central part of the many-nanoparticle chain whereas at the chain edges the interparticle distance exceeds one wavelength by several percent. He also studied the field enhancement in such a chain and showed that the dipole in the middle of the chain feels increasing field only up to a certain number of dipoles in the chain; if this critical number is exceeded, the field decreases together with the trapping potential. To avoid this phenomenon for chains with more particles he reduced the coherence of the laser light. However, the field enhancement was not as large as for the case of the spatially modulated phase of the incoming light.

Particle binding in broadband light sources was considered by Rodriguez and Andrews (2009a, 2009b) using the QED-cast method of induced moments. They showed that the distance between the particles in the chain can be tuned by filtering the broadband radiation. The less coherent the source, the smaller the interparticle distance in the deepest (first) stable configuration.

de Abajo (2007) recently presented an extensive work concerning light scattering by particles and holes arranged in planar periodic arrays. He focused mainly on the reflectance or transmittance of such systems close to the surface, considered resonance conditions, and the influence of surface plasmons. However, he had not dealt with the force interactions between the illuminated objects.

\section{Dipole near a planar surface}

The study of dipole radiation near a surface or planar layered media has found many useful applications in the field of microscopy, single molecule spectroscopy, cavity quantum electrodynamics, integrated optics, and surface enhanced Raman spectroscopy above metallic surfaces. Here we follow the theoretical treatment of Novotny and Hecht (2006) using the dyadic Green's function formalism. Consider first a single dipole placed at $\mathbf{r}_{0}$ (distance $z_{0}$ above the surface). Due to the presence of the surface the field at the position $\mathbf{r}$ is given by

$$
\mathbf{E}(\mathbf{r})=\mathbf{E}^{I}(\mathbf{r})+\left[\overline{\mathbf{G}}\left(\mathbf{r}, \mathbf{r}_{0}\right)+\overline{\mathbf{G}}_{\mathrm{ref}}\left(\mathbf{r}, \mathbf{r}_{0}\right)\right] \bar{\alpha} \mathbf{E}\left(\mathbf{r}_{0}\right),
$$

where $\overline{\mathbf{G}}\left(\mathbf{r}, \mathbf{r}_{0}\right)$ denotes the dyadic Green's function in free space used in Sec. III.A [Eq. (4)] and $\overline{\mathbf{G}}_{\text {ref }}\left(\mathbf{r}, \mathbf{r}_{0}\right)$ denotes the dyadic Green's function due to the dipole radiation reflection at the surface. Using the spectrum of plane waves reflected from the surface the general form for the field was found (Novotny, 1997a, 1997b; Novotny and Hecht, 2006). Unfortunately, no closed solution ex- 
ists for this problem without using certain approximations.

Chaumet and Nieto-Vesperinas (2000b) studied the geometry of a single dipolar particle placed in a low refractive index medium below a dielectric interface and gradually extended this problem to a metal nanoparticle (Chaumet and Nieto-Vesperinas, 2000c). Their analyses of the metal nanoparticles revealed different behavior if the trapping laser wavelength was below the plasmon resonance frequency. In this case, the particle polarizability reverses its sign and the gradient force repels the particle out of high-intensity regions. They also considered evanescent wave (EW) illumination when the angle of the beam incident upon the surface is larger than the critical angle.

Optical binding of two particles below the interface has also been studied (Chaumet and Nieto-Vesperinas, 2001; Nieto-Vesperinas et al., 2004) but with no remarkable deviations from the binding far from the surface. In general, the influence of the surface is not dominant if the particle center is further away from the surface than its diameter (Arias-González and Nieto-Vesperinas, 2003).

\section{B. Optical binding of micro-objects}

The theoretical description of the interaction between larger particle (micro-objects) mediated by light is much more complex than that used to describe nanoparticles. However, it is important to extend our understanding of the optical binding at this scale because micro-objects are more closely aligned to typical experimental observations and thus facilitate direct and needed comparisons between experiment and theory.

\section{Optical forces}

The optical force is the result of the transfer of momentum from the light to a micro-object. Two general theoretical approaches are typically adopted to express such a force. One of these approaches builds on the fact that an object illuminated by an incident light wave scatters this light and, thus, modifies the final light distribution. From the overall momentum balance, the timeaveraged optical force acting on the illuminated object is given by (Stratton, 1941)

$$
\langle\mathbf{F}\rangle=\frac{1}{2} \operatorname{Re}\left\{\oint_{S}\langle\mathbf{T}\rangle \cdot \mathbf{n} d S\right\},
$$

where the integration is performed over an arbitrary closed surface $S$ enclosing the object and $\mathbf{n}$ is the outer unit normal to the surface $S$. The components of the stress tensor $\overline{\mathbf{T}}$ have the following form in a linear and isotropic medium:

$$
\begin{aligned}
T_{i j}= & \varepsilon_{0} \varepsilon_{m} E_{i} E_{j}^{*}+\mu_{0} \mu_{m} H_{i} H_{j}^{*} \\
& -\frac{1}{2}\left(\varepsilon_{0} \varepsilon_{m}|E|^{2}+\mu_{0} \mu_{m}|H|^{2}\right) \delta_{i j},
\end{aligned}
$$

where $|E|$ and $|H|$ is the magnitude of the electric and magnetic field vector, $\varepsilon_{0}$ and $\mu_{0}$ are the permittivity and permeability of vacuum, and $\varepsilon_{m}$ and $\mu_{m}$ are the relative permittivity and permeability of the medium surrounding the object, respectively. The field components entering Eq. (36) correspond to the total field (i.e., incident plus scattered) outside the object. No material properties of the object are present explicitly in Eq. (35); however, they are hidden in the methods for calculating these fields. Integration over an arbitrary surface enclosing the studied object is the largest computational advantage of this method that facilitates analytical treatment in some cases. The scattered field can be expressed analytically only for a limited number of symmetries of the object. The most frequently treated shape is a sphere where Mie scattering theory is used (Barton et al., 1989; Ren et al., 1996; Mazolli et al., 2003; Rohrbach, 2005; Neves et al., 2007; Viana et al., 2007). A group of spheres can be treated using the multiple Mie scattering theory (Xu, 1995; Ng, Lin, et al., 2005). In addition to spherical objects, spheroidal or cylindrical ones are also often considered theoretically (Nieminen et al., 2001; Grzegorczyk et al., 2006c; Nieminen, Kröner, et al., 2007; Xu et al., 2007).

Optical forces acting on particles with more complex shapes must be calculated using numerical schemes; for example, the coupled dipole method (CDM) (Hoekstra et al., 2001; Chaumet and Billaudeau, 2007), finite element method (White, 2000), or finite-difference timedomain (FDTD) method (Collett et al., 2003; Gauthier, 2005; Benito et al., 2008). A MATLAB toolbox is available for the calculation of optical forces acting on spherical and spheroidal objects in Gaussian and other beams (Nieminen, Loke, et al., 2007). If the object is much larger than the trapping light wavelength, the ray-optics model can be used (Ashkin, 1992; Gussgard et al., 1992; Gu et al., 1997; Mazolli et al., 2003).

The second approach derives the optical force from the Lorentz force acting on both currents $\mathbf{J}$ due to the polarization of dielectric and bound charges $\rho_{e}$ at the boundaries (Mansuripur, 2004, 2005; Kemp et al., 2005, 2006),

$$
\langle\mathbf{F}\rangle=\frac{1}{2} \operatorname{Re}\left\{\int\left\langle\rho_{e} \mathbf{E}^{*}+\mathbf{J} \times \mathbf{B}^{*}\right\rangle d V\right\} .
$$

The integration in Eq. (37) is performed over the volume of the particle. This method usually requires more tedious numerical integration but can also be easily integrated into FDTD calculations (Zakharian et al., 2005, 2006). In contrast to the stress tensor method, the Lorentz force approach can calculate the distribution of the force density inside the object.

Both methods can be directly applied for the calculation of optical binding of micro-objects; however, various numerical approaches must be used based on the symmetry of the studied object.

\section{Optical binding of spheres}

This topic has been explored in depth by $\mathrm{Ng}$, Lin, et al. (2005) who assumed a configuration with two coun- 
terpropagating linearly polarized plane waves forming a standing wave with steep intensity gradients between the interference maxima and minima. This configuration localizes the particles in an intensity maximum or minimum according to their size (Zemánek et al., 2002). Hence the particle behavior can be treated as quasi-twodimensional in the plane perpendicular to the propagation direction of the plane waves. The work of $\mathrm{Ng}$, Lin, et al. is the first example of a detailed theory for lateral 2D binding of microspheres. They calculated the timeaveraged force based on Eq. (35) and expressed the electromagnetic field around the particles using accurate multiple-scattering theory (Xu, 1995). A detailed theoretical description of this method is beyond the extent of this paper; it is possibly the most accurate and efficient way in which the optical binding of multiple spherical particles can be treated. In the following, we present some of the major results with their method.

They assumed that the calculated optical force acting upon the sphere can be fitted asymptotically to

$$
F_{\xi} \simeq \frac{f_{\xi}(a)}{R} \cos [k R+\Phi(a)],
$$

where $\xi$ denotes the lateral components (here $x, y$ ) of the optical force, $f_{\xi}(a)$ denotes the strength (amplitude) of the optical force, and $\Phi(a)$ represents the fitted phase. They showed that there exist several stable positions along the $x$ axis (in parallel with the light polarization) separated by about a wavelength. However, if the bisphere axis is oriented perpendicular to the light polarization, only one stable position exists for touching spheres, which is exactly opposite behavior as described for nanoparticles (see Fig. 6) caused by the selected size of the microspheres.

Optical binding between spheres can be greatly enhanced if they are close to one another and one exploits so-called morphology-dependent resonances (MDRs) (Ng, Chan, et al., 2005; Povinelli, Johnson, et al., 2005). Both attractive and repulsive binding forces were found depending on whether the resonance mode of the two bound spheres is symmetric or antisymmetric. The forces are linearly enhanced by the quality of the MDR resonator. $\mathrm{Ng}$, Chan, et al. (2005) focused on a single plane wave propagating along the bisphere axis. They showed that even if the radiation pressure on a single sphere is increased by just $30 \%$ and less due to MDR, the binding force increase between the spheres can be significantly higher especially for size parameters $k a$ between 20 and 30 .

\section{Paraxial optics theory for larger spheres}

The paraxial optics approach is an approximation to the full vectorial Maxwell theory that provides a framework for longitudinal optical binding of larger particles. Here the dominant binding mechanism is light refocusing by the trapped particles, for example, dielectric spheres or cells. More specifically, the paraxial theory applies to particles whose diameter is greater than the optical wavelength, and trapping beams that are not too tightly focused, thereby allowing for a scalar paraxial treatment of the field propagation and small refractive index differences between the spheres and host liquid so that back reflections may be neglected to leading order. In this limit the paraxial theory accurately captures the refocusing of the binding laser beams due to the presence of the particles and provides a useful paradigm for longitudinal optical binding based on refocusing. In comparison to the more general vector Maxwell theory, the paraxial theory has the virtues that it is considerably less computer intensive, thereby allowing for treatment of longitudinal optical binding over spatial scales much larger than the wavelength. Naturally, of course, this has limitations in that we cannot really deal with large refractive index changes between the particle and the surrounding medium and of course we may lose some of the detailed physics of the situation.

For longitudinal optical binding we consider two monochromatic laser beams of frequency $\omega$ counterpropagating along the $z$ axis which interact with a system of $N$ transparent dielectric spheres of mass $m$, refractive index $n_{p}$, and radius $a$, with centers at positions $\left\{\mathbf{r}_{j}(t)\right\}, j=1,2, \ldots, N$, and which are immersed in a host medium of refractive index $n_{m}$. The monochromatic electric field component $E_{i}$ is expressed as a sum of the positive and negative frequency components,

$$
E_{i}(\mathbf{r})=E_{i+}(\mathbf{r}) \exp (i k z)+E_{i-}(\mathbf{r}) \exp (-i k z),
$$

where $\mathbf{E}_{ \pm}(\mathbf{r})$ are the slowly varying electric field amplitudes of the right or forward propagating $(+)$ and left or backward propagating $(-)$ fields. Any paraxial incident fields can be considered, e.g., collimated Gaussian beams.

Consider first that the dielectric spheres are in a fixed configuration at time $t$ specified by the centers $\left\{\mathbf{r}_{j}(t)\right\}$. Then the dielectric spheres provide a spatially inhomogeneous refractive index distribution of the form

$$
n^{2}(\mathbf{r})=n_{m}^{2}+\left(n_{p}^{2}-n_{m}^{2}\right) \sum_{j=1}^{N} \theta\left(a-\left|\mathbf{r}-\mathbf{r}_{j}(t)\right|\right),
$$

where $\theta\left(a-\left|\mathbf{r}-\mathbf{r}_{j}(t)\right|\right)$ is the Heaviside step function which is unity within the sphere of radius $a$ centered on $\mathbf{r}=\mathbf{r}_{j}(t)$ and zero outside. Then, following standard procedures, the counterpropagating fields evolve according to the paraxial wave equations

$$
\pm \frac{\partial E_{i \pm}}{\partial z}=\frac{i}{2 k} \nabla_{\perp}^{2} E_{i \pm}+i k_{0} \frac{n^{2}(\mathbf{r})-n_{m}^{2}}{2 n_{m}} E_{i \pm},
$$

where $k_{0}=\omega / c$ and $\nabla_{\perp}^{2}=\partial^{2} / \partial x^{2}+\partial^{2} / \partial y^{2}$ is the transverse Laplacian describing the beam diffraction. A given configuration of the dielectric spheres modifies the fields $E_{i \pm}(\mathbf{r})$ in a way that can be calculated from the above field equations. Even in the case the spheres move and hence the refractive index distribution varies, the fields will always adiabatically slave to the instantaneous sphere configuration. This paraxial wave theory has been validated against experiment for propagation through a system of dielectric spheres using a femto- 
second laser source and adding fluorescein to the host liquid. The field intensity profile could be measured by detecting the associated two-photon fluorescence (Dholakia et al., 2004; Metzger, Wright, Sibbett, and Dholakia, 2006).

McGloin et al. (2004) applied the paraxial field propagation method to study the longitudinal optical binding of two and more spheres placed into counterpropagating incoherent Gaussian beams with aligned centers $z=-L / 2$ for the forward field and $z=L / 2$ for the backward field, where $L$ denotes the distance between beam waists. The Gaussian input beams supply strong enough transverse confinement that the sphere motion remains directed along the $z$ axis and only the $z$ component of the force is of interest. To calculate the forces acting on the spheres they followed the approach of Zakharian et $a l$. and expressed the cycle-averaged force acting on the $j$ th dielectric using the Lorentz force law [Eq. (37)]. The first term in the integrand $\rho_{e} \mathbf{E}^{*}$ gives rise to a force acting along the direction of polarization of the laser fields and perpendicular to the $z$ axis, and for the present configuration it is not relevant. Using the paraxial form of the field from Eq. (39) the second term in Eq. (37) gives the force acting upon particle $j$ in the system of dielectric spheres with the optical polarization $\mathbf{P}(\mathbf{r}, t)=\epsilon_{0}\left[n_{p}^{2}(\mathbf{r})\right.$ $\left.-n_{m}^{2}\right] \mathbf{E}(\mathbf{r}, t)$ and the current density due to bound charges $\mathbf{J}_{b}=\partial \mathbf{P} / \partial t$,

$$
\begin{aligned}
F^{(j)}(t) & =\frac{\epsilon_{0}\left(n_{p}^{2}-n_{m}^{2}\right)}{4} \int_{V^{(j)}} d^{3} \mathbf{r}^{\prime}\left(\frac{\partial\left|E_{+}\right|^{2}}{\partial z^{\prime}}+\frac{\partial\left|E_{-}\right|^{2}}{\partial z^{\prime}}\right) \\
& =\frac{\epsilon_{0}\left(n_{p}^{2}-n_{m}^{2}\right)}{4 k} \int_{V^{(j)}} d^{3} \mathbf{r}^{\prime} \operatorname{Im}\left(E_{-}^{*} \nabla_{\perp}^{2} E_{-}-E_{+}^{*} \nabla_{\perp}^{2} E_{+}\right) .
\end{aligned}
$$

The final expression in Eq. (42) is used in the simulations to determine numerically the force on each sphere; in general, this force is a combination of both gradient and scattering forces. This method has been used for investigation of optical binding of two and three spheres and successfully compared to experimental results of longitudinal optical binding (Metzger, Dholakia, and Wright, 2006; Metzger, Wright, and Dholakia, 2006).

\section{Optical binding of infinite cylinders}

Grzegorczyk et al. (2006c) focused on the geometry of infinite cylinders and used the exact Foldy-Lax multiplescattering equations to properly describe the interactions between many particles (Foldy, 1945; Lax, 1951, 1952). For the sake of simplicity they arranged the incoming wave vectors perpendicular to the axes of the cylinders and as a result could treat arbitrary number of cylinders of arbitrary sizes without an additional approximation. However, the disadvantage was that all particles had to be cylinders of identical permittivity. They calculated the electromagnetic field around the cylinders using the multiple-scattering theory and obtained the optical forces from the Maxwell stress tensor (35). The force along the $x$ axis for various cylinder con-

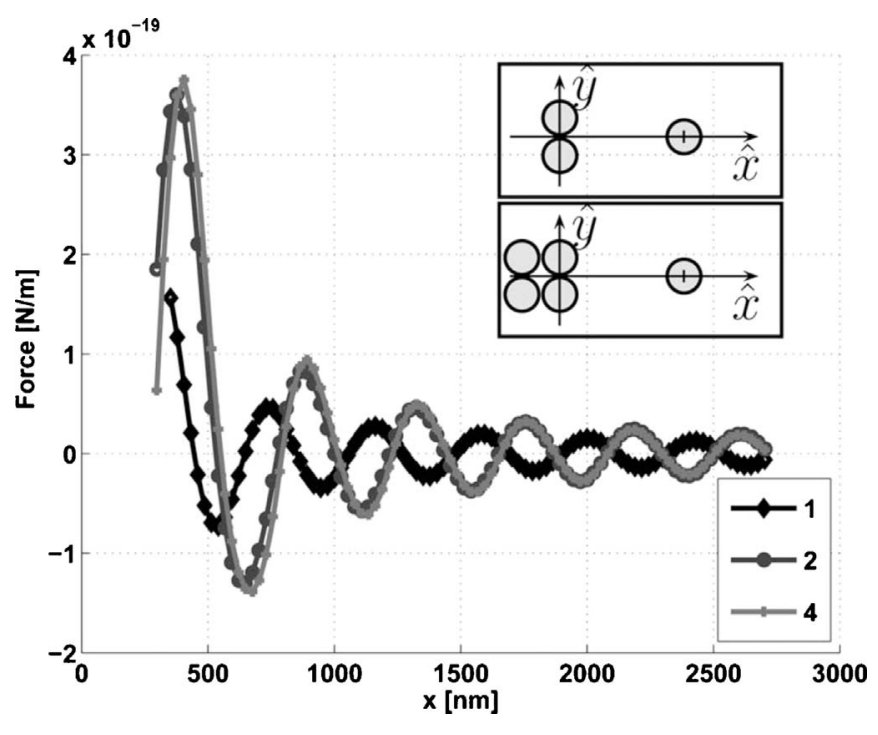

FIG. 8. Optical binding of infinite cylinders under plane-wave illumination. Force along the $x$ axis (light propagation direction) acting on the right-hand side cylinder for a varying number of cylinders on the left. Case 1 corresponds to two cylinders; cases 2 and 3 are explained in the inset. The parameters used are the following: cylinder radius $a=0.3 \lambda$, laser wavelength $\lambda=546 \mathrm{~nm}$, permittivity of polystyrene cylinder $\varepsilon_{p}$ $=2.56$, and water $\varepsilon_{m}=1.69$. From Grzegorczyk et al., 2006c.

figurations is again periodic with a period close to the light wavelength (see Fig. 8). In the considered cylinder configurations, the majority of the cylinders are fixed and create a diffractive structure scattering the incident light toward one free cylinder spanning the space (Grzegorczyk et al., 2006a, 2006b, 2006c). Figure 8 shows that the force acting on the free cylinder increases with the number of fixed cylinders arranged along the $y$ axis.

Using the side scattering arrangement and plane-wave illumination along the $y$ axis, Grzegorczyk et al. (2006a) suggested optical guiding, sorting, and even optical confinement between two arrays of fixed cylinders (Grzegorczyk et al., 2006b). Their model also enabled them to treat the mutual interactions between cylinders if illuminated with spatially structured beams; for example, three interfering plane waves. Figure 9 shows this model with 20 dielectric cylinders placed in three-plane-wave interference pattern.

\section{Optical binding using the coupled dipole method}

The coupled dipole approximation, also known as discrete dipole approximation (Purcell and Pennypacker, 1973; Draine, 1988), is based on the decomposition of the object into $N$ dipoles placed in an orthogonal grid with interdipole distances $d$ so small that the object shape is described satisfactorily and the field in the vicinity of each dipole can be considered uniform $(|m| k d$ $\leqslant 1$, where $m$ is the ratio of refractive indices of the object and the surrounding medium and $k$ is the wave number). Based on Eqs. (3) a linear system of coupled equations describes the $i$ th component of the total field $E_{i}^{\xi}$ at the position of dipole $\xi$. However, $N$ in the order 

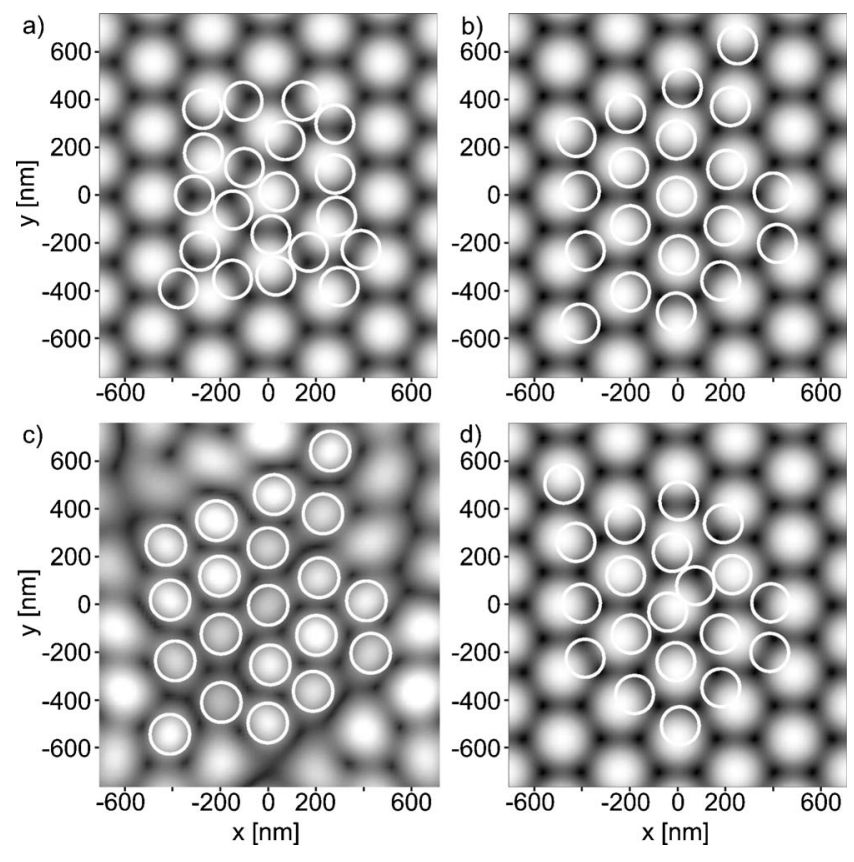

FIG. 9. Optical binding of infinite cylinders in the interference field of plane waves. Positions of 20 cylinders (white circles) and field distributions (background gray-scale images) for (a) random initial positions in a three-plane-wave interference pattern, background shows unperturbed incident field distribution; (b) organized final positions due to the trapping and binding forces, background shows unperturbed incident field distribution; (c) the same as (b) but final field distribution is shown at the background; and (d) organized final positions corresponding to another set of cylinders in initial positions. The parameters used are the following: $a=0.15 \lambda, \lambda=546 \mathrm{~nm}, \varepsilon_{p}$ $=2.56$, and $\varepsilon_{m}=1.69$. Adapted from Grzegorczyk et al., 2006c.

of thousands to millions is usually needed to obtain exact results for micro-objects. Therefore iterative biconjugate gradient methods must be used with the number of numerical operations $\simeq N^{2}$ (Press et al., 1992). Another problem is the computer memory requirement ( $\simeq 144 N^{2}$ bytes) to allocate the matrices of the necessary Green's functions. Fortunately if the dipoles are situated on a periodic lattice, a fast Fourier transform algorithm is applicable and it further decreases the number of numerical operations to $N \log N$ (Goodman et al., 1991; Draine and Flatau, 1994; Hoekstra et al., 2001). For large $N$ this is an important breakthrough.

Once the dipole moments of all dipoles in the system are known, the final electric field components at the positions of each dipole can also be determined using Eq. (7) and one can express the force acting on each dipole. The force acting on the whole solid object $B$ is done as the sum of the forces acting on all dipoles forming this object,

$$
F_{i}^{B}=\sum_{\nu \in B} F_{i}^{\nu}
$$

The time-averaged optical forces $\langle\mathbf{F}\rangle$ acting on an object can also be obtained using the stress tensor (35) with the field components obtained from the CDM where the double integration over the closed surface must be performed numerically. Both methods based on Eqs. (43) and (35) give similar results that differ in units of percent from the exact forces obtained from the Mie approach (Chaumet and Nieto-Vesperinas, 2000b). The principal advantage of the CDM is its applicability to general object shapes and composition and its easy extension to the optical torque calculations (Draine and Flatau, 1994; Draine and Weingartner, 1996; Hoekstra et al., 2001; Chaumet and Billaudeau, 2007). It has been shown that the CDM is equivalent to the digitized Green's function method or volume-integral equation formulation in the low-frequency limit $k d \rightarrow 0$ (Draine and Flatau, 1994). The accuracy of the CDM suffers if the object refractive index is high compared to the refractive index of the surrounding medium. In this case it is preferred to use a more complex description of the polarizability of the dipolar units (dipoles) (Chaumet et al., 2004).

Since the CDM inherently contains the interaction of a selected dipole with all other dipoles in the system, splitting one object into several objects provides an easy way to treat optical binding between them by the CDM (Karásek, Dholakia, and Zemánek, 2006; Karásek et al., 2009). It was shown (Karásek, Dholakia, and Zemánek, 2006) how minute variations of the Gaussian beam waist radius by only $200 \mathrm{~nm}$ or particle diameter by $40 \mathrm{~nm}$ or object refractive indices by just $\simeq 1 \%$ can cause the collapse of a stable two-particle longitudinally bound structure or change the interparticle distance by several micrometers. Since the CDM incorporates the backwardscattered waves, in contrast to the paraxial approach the CDM predicts correctly the existence of several equilibrium positions for each stable two-particle structure separated from each other by half a wavelength. This multistability comes from the interference of counterpropagating waves explained for longitudinal binding of dipoles. However, for larger objects examined here it leads to the oscillations modulated on a more complex force background. Karasek et al. also predicted that the amplitude of the force oscillations depends on the size of the bound objects (Karásek, Čižmár, and Zemánek, 2006) and for certain particle diameters they even disappear. If the scattering object is so large that the backward-scattered field is suppressed (within the CDM model this happens due to the overall destructive interference from the dipoles in the scattering object), the net force coming from the interaction of this field with any dipole forming the second object will be suppressed too.

The CDM can serve as a universal tool for direct comparison of various particle shapes or configurations with experimental observations. For example, comparison of the CDM results with experimental observations of longitudinal binding (Metzger, Dholakia, and Wright, 2006) gave excellent coincidence taking into account the spread of the experimental values. It even confirmed the observed bistability of interparticle distances induced by the refractive index variations of the surrounding medium (Karásek, Dholakia, and Zemánek, 2006). The CDM was also applied in the study of longitudinal binding in "nondiffracting" beams (Durnin et al., 1987). 


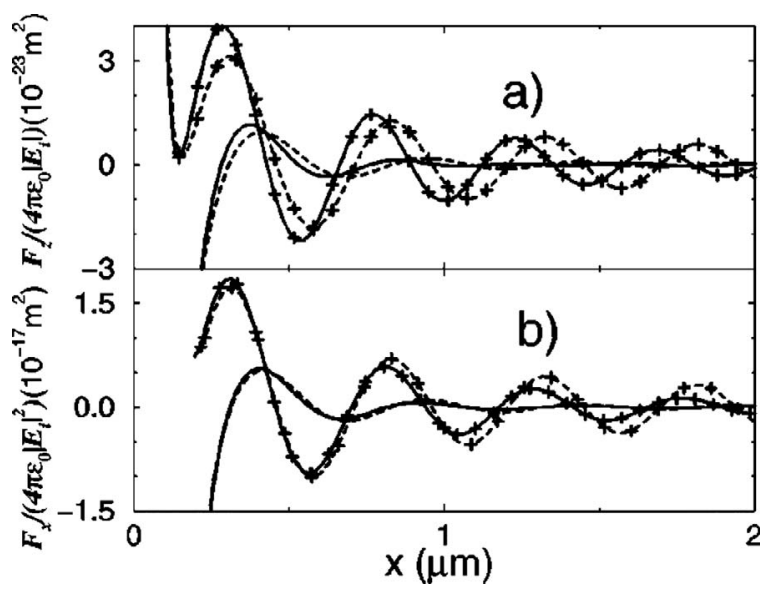

FIG. 10. Optical binding of spheres near a planar dielectric interface. Two glass spheres are placed at the same distance below the dielectric interface oriented along the $x$ axis. The incident beam $(\lambda=632.8 \mathrm{~nm})$ impinges perpendicularly to the interface and illuminates both spheres. The binding forces shown between these spheres for $s$ (marked + ) and $p$ polarizations. Full curves denote the forces in the presence of the surface and dashed curves without it. Glass spheres have radii of (a) $10 \mathrm{~nm}$ and (b) $100 \mathrm{~nm}$. From Chaumet and NietoVesperinas, 2001.

These beams do not change their lateral profiles while they propagate and, thus, longitudinal optical binding is not influenced by the intensity variations of the axial beam profile. Hence the interparticle interactions via light scattering become the dominant mechanism responsible for the particle arrangement (Karásek and Zemánek, 2007; Karásek et al., 2008, 2009). The CDM also enables, within the technical limitation of the used computers, to treat optical binding of more objects. This case was studied again for longitudinal binding in the Bessel beams (Karásek et al., 2008) and the calculated data were compared with the experimental results with good agreement including the observation of the shortand long-range self-arranged chains of particles (see Fig. 12).

\section{Optical binding near a flat surface}

The description of an induced dipole radiation near a surface introduced in Sec. III.A.6 can be extended using the CDM to larger objects. Chaumet and NietoVesperinas used such an approach to calculate the optical forces acting on a sphere placed close to a surface (Chaumet and Nieto-Vesperinas, 2000b) and on a pair of spheres with and without the presence of a surface (Chaumet and Nieto-Vesperinas, 2001). As shown in Fig. 10 the presence of a surface influences the binding in the considered configuration only weakly for larger objects. For smaller spheres there is a shift in the equilibrium distances between the spheres reaching hundreds of nanometers. Behavior of two and three metallic nanoparticles in various configurations near a surface was investigated by Zelenina et al. (2007). They showed enhanced near-field binding forces for nonlinear arrangement of the particles and for the wavelength close to the plasmon resonance. Stronger binding forces were observed for three particles in a nonlinear arrangement.

Numerical approaches are more suitable to describe the real experimental situations. The finite element method and commercial software (COMSOL) (Gaugiran et al., 2005, 2007) or finite-difference time-domain method (Benito et al., 2008) express the electromagnetic field around the particles and the optical forces are then calculated using Eq. (35). However, there have been relatively few results in this area.

\section{Dynamical phenomena in optical binding of micro-objects}

\section{Particle dynamics due to optical forces}

The existence of a stable configuration of particles with zero force acting on each of them does not ensure the overall long-term stability of the cluster and, indeed, we need to consider that these are dynamically evolving systems due to the presence of fluid and hydrodynamics for example. The dynamical behavior must be treated using the equation of motion of the individual particles to obtain the stability criteria. $\mathrm{Ng}$, Lin, et al. (2005) treated the problem of optically bound microspheres in two dimensions in a general way. They considered only the influence of the optical forces and omitted the hydrodynamic interactions and the stochastic thermal activation. To describe the particle positions, they used general position vector $\tilde{\mathbf{r}}^{\text {eq }}$ $=\left(\tilde{r}_{1}^{\mathrm{eq}}, \tilde{r}_{2}^{\mathrm{eq}}, \ldots, \tilde{r}_{2 N-1}^{\mathrm{eq}}, \tilde{r}_{2 N}^{\mathrm{eq}}\right)$. Coordinate pairs $\tilde{r}_{2 i-1}, \tilde{r}_{2 i} \mathrm{de}-$ scribe the position of $i$ th particle in the lateral plane $\left(x_{i}, y_{i}\right)$. The linearized equation of motion at $\tilde{\mathbf{r}}^{\text {eq }}$ then has the following form:

$$
m \frac{d^{2} \Delta \tilde{r}_{j}}{d t^{2}} \approx \tilde{K}_{j k} \Delta \tilde{r}_{k}-b \frac{\Delta \tilde{r}_{j}}{d t}
$$

where $m$ is the spheres mass, $\Delta \tilde{r}_{j}=\tilde{r}_{j}-\tilde{r}_{j}^{\text {eq }}$ denotes the displacement of $j$ th component of general position vector of the spheres $\tilde{r}_{j}$ from its local equilibrium component $\tilde{r}_{j}^{\mathrm{eq}}, b$ is the viscous damping constant, and the stiffness tensor $\tilde{K}_{j k}$ satisfies

$$
\tilde{K}_{j k}=\frac{\partial \tilde{F}_{j}}{\partial \Delta \tilde{r}_{k}}
$$

where $\tilde{F}_{j}$ is the $j$ th component of the general optical force vector constructed in the same way as $\tilde{\mathbf{r}}$.

It is assumed above that the equilibrium is created by the zero force acting upon each sphere (the so-called static equilibrium). However, $\mathrm{Ng}$, Lin, et al. (2005) showed that a special type of equilibrium called drifting equilibrium also exists. In this case the forces on each sphere are not equal to zero but are constant and identical [cf. Eq. (29)]. Therefore the spheres keep their distances fixed but the whole formation (cluster) moves while keeping its shape. Dynamic equilibrium is a more generalized description requiring an extra additive term at the right-hand side of Eq. (44). Such an equation, 


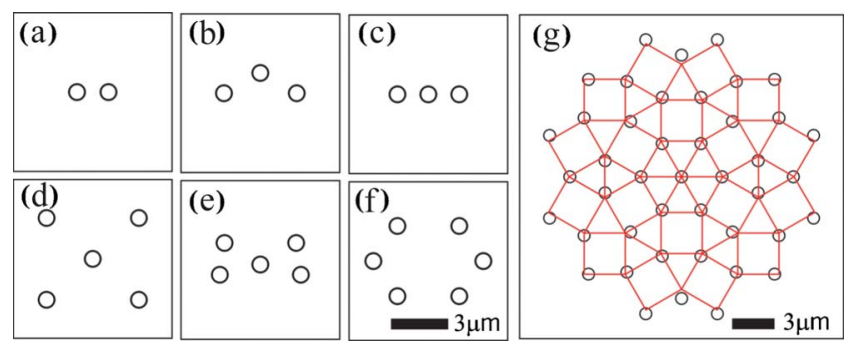

FIG. 11. (Color online) Stability of optical binding of multiple identical spheres. Examples of equilibrium configurations calculated for varying numbers of polystyrene $\left(\varepsilon_{p}=2.53\right)$ spheres of radius $a=0.414 \mu \mathrm{m}$ placed in vacuum (air) and illuminated with horizontal polarization of the incident light with a wavelength $\lambda=0.52 \mu \mathrm{m}$. Configurations (a)-(e) have all eigenmodes stable, (b) and (e) are in drifting equilibrium, and (f) and (g) have either stable or quasistable modes (see the text). From $\mathrm{Ng}$, Lin, et al., 2005.

however, can be transformed back to the form of Eq. (44). Using the coordinate transform $\Delta \tilde{r}_{j}=V_{j k} \eta_{k}, \mathrm{Ng}$, Lin, et al. (2005) decoupled Eq. (44) into

$$
m \frac{d^{2} \eta_{j}}{d t^{2}}=\tilde{K}_{j} \eta_{j}-b \frac{\eta_{i}}{d t}
$$

where $V_{j k}$ for $k=1, \ldots, 2 N$ are eigenvectors of $\tilde{\mathbf{K}}$ so that $\tilde{\mathbf{K}}$ is diagonalized with eigenvalues $\tilde{K}_{i}$. The expected solution of Eq. (46) has the form $\eta_{j}=\eta_{j 0} \exp \left(i \Omega_{j 0} t\right)$ where the natural frequency of the eigenmode is given by $\Omega_{j 0}$ $=\sqrt{-\tilde{K}_{j} / m}$. Therefore the eigenvalues $\tilde{K}_{i}$ dictate the stability of the optically bound cluster of particles. The general solution of Eq. (46) is obtained as a linear combination of neutral, unstable, stable, complex unstable, and quasistable modes. A cluster is called stable if all its eigenmodes are neutral or stable. However, the stable cluster formations found by the linear analyses must also be stable against the thermal fluctuations occurring in real experiments. This requires that dissociation energy per sphere is much higher than $k_{B} T$ ( $k_{B}$ being the Boltzmann constant and $T$ being the absolute temperature). An unstable cluster is formed if one unstable or complex unstable mode is present. Finally, a cluster is termed quasistable if it consists of neutral, stable, and quasistable mode(s). Quasistable clusters can be locked into a periodic orbit if appropriate friction is present but their behavior can only be studied by molecular dynamics. $\mathrm{Ng}$, Lin, et al. (2005) presented a detailed theoretical description and numerous results. Figure 11 shows examples of static and drifting equilibria together with stable and quasistable clusters.

$\mathrm{Ng}$ and Chan (2006) also studied a different configuration of a linear array of $N$ spheres confined in a standing wave formed from two counterpropagating plane waves. Each of the particles occupied one intensity maximum in the standing wave where it was confined by the gradient forces but laterally their motion was dictated by the optical binding. They showed that the potential energy of each sphere in the array could increase by $20 \%$ due to the contribution of the binding force as the number of spheres in the array increased from 1 to 100. Even though the field intensity did not vary in the lateral plane, the equally spaced particles formed a linear array with three distinct vibration mode branches. The best sphere localization and the highest sustained vibrational frequencies were provided in the vibration along the beam propagation direction and were caused by the standing-wave traps. In this configuration, optical binding stabilized the lateral motion of the objects. Lateral vibrations in the direction parallel or perpendicular to the field polarization were less localized with lower vibrational frequencies.

\section{Hydrodynamic equations}

The optical forces derived above are useful to calculate the equilibrium positions of the particles for which the optical forces $\mathbf{F}_{j}=0$. However, full equations of particle motion are required to deal with transient or noisedriven phenomena. In general the motion of the particles in the host fluid will be overdamped and hydrodynamics interactions will influence the particle dynamics in addition to the optical forces (Metzger et al., 2007). In the overdamped limit the equation of motion for the spheres is

$$
\mathbf{v}_{j}=\frac{d \mathbf{r}_{j}}{d t}=\sum_{k=1}^{N} \mathbf{H}_{j k} \mathbf{F}_{k}+\mathbf{f}_{j}(t),
$$

with the Oseen tensor $\mathbf{H}$ given by

$$
\mathbf{H}_{j k}=\frac{\mathbf{I}}{b} \delta_{j k}+\frac{3 a}{4 b r_{j k}}\left(1-\delta_{j k}\right)\left(\mathbf{I}+\frac{\mathbf{r}_{j k} \mathbf{r}_{j k}}{r_{j k}^{2}}\right),
$$

where $\mathbf{I}$ is the unit second rank tensor, $\mathbf{r}_{j k}=\mathbf{r}_{j}-\mathbf{r}_{k}$, and $b=6 \pi \eta a, \eta$ being the viscosity of the host medium. The Oseen tensor accounts for cross coupling between the particle motions due to hydrodynamic forces, and $\mathbf{f}_{j}(t)$ are randomly fluctuating vector functions representing the fluctuating forces acting on the particles due to Brownian motion.

The above general formulation can be greatly simplified to treat the correlations that exist between two optically bound particles with equilibrium separation $R$. In this case the random forces associated with the Brownian motion will drive small motions that can become correlated by optical binding forces and hydrodynamic interactions (Meiners and Quake, 1999; Bartlett et al., 2001). The two identical spheres are assumed to be longitudinally optically bound along the $z$ axis due to illumination by a pair of mutually incoherent but otherwise identical counterpropagating laser fields in a dual beam fiber trap. We label the deviations of the sphere centers from their equilibrium positions along the $z$ axis by $z_{j}(t)$, $j=1,2$, and assume that the spheres are tightly bound in the plane transverse to the laser propagation axis due to the confinement provided by the Gaussian intensity profiles. Thus, we can concentrate on the longitudinal particle motions along the $z$ axis. Adopting the notation of 
Bartlett et al. (2001) for small sphere displacements Eq. (47) can be reduced to the matrix Langevin equation,

$$
\frac{d}{d t}\left(\begin{array}{l}
z_{1} \\
z_{2}
\end{array}\right)=\left(\begin{array}{ll}
A_{11} & A_{12} \\
A_{12} & A_{11}
\end{array}\right)\left(\begin{array}{l}
f_{1}(t)-k z_{1}+\xi z_{2} \\
f_{2}(t)-k z_{2}+\xi z_{1}
\end{array}\right),
$$

where $A_{12}=1 / 6 \pi \eta a=\epsilon A_{11}, \epsilon=3 a /(2 R)$ describe the longitudinal particle mobilities and the fluctuating forces $f_{j}(t)$ have zero mean $\left\langle f_{i}(t)\right\rangle=0$ and correlation functions $\left\langle f_{j}(t) f_{k}\left(t^{\prime}\right)\right\rangle=2\left(A^{-1}\right)_{j k} k_{B} T \delta\left(t-t^{\prime}\right)$ at temperature $T$. The force terms proportional to the spring constant $k>0$ represent the restoring forces on a sphere chosen when that sphere is displaced while the other sphere is held fixed, and the force terms proportional to $\xi>0$ describe the cross force acting on the sphere chosen at its equilibrium position when the other sphere is displaced.

To facilitate the analysis of the matrix Langevin equation [Eq. (49)] normal mode coordinates $Z_{1}=\left(z_{1}+z_{2}\right) / 2$ for the center-of-mass motion and $Z_{2}=z_{1}-z_{2}$ for the relative motion may be used. Then applying the methods described by Bartlett et al. (2001), Metzger et al. (2007) found the normal mode correlation functions

$$
C_{j}(t)=\frac{\left\langle Z_{j}(t) Z_{j}(0)\right\rangle}{\left\langle Z_{j}^{2}(t)\right\rangle}=\exp \left(-\frac{|t|}{\tau_{j}}\right),
$$

where $t$ is the delay time and the decay times $\tau_{j}$ for the center-of-mass $(j=1)$ and relative $(j=2)$ normal modes are given by

$$
\begin{aligned}
& \frac{1}{\tau_{1}}=k A_{11}(1+\epsilon)\left[1-\frac{\xi}{k}\right], \\
& \frac{1}{\tau_{2}}=k A_{11}(1+\epsilon)\left[1+\frac{\xi}{k}\right] .
\end{aligned}
$$

Experimentally the decay times $\tau_{1,2}$ can be measured and the above formulas can then be combined to yield

$$
\frac{\xi}{k}=\frac{(1+\epsilon) /(1-\epsilon)-\tau_{2} / \tau_{1}}{(1+\epsilon) /(1-\epsilon)+\tau_{2} / \tau_{1}} .
$$

Since $\epsilon$ is known, the force constants $k$ and $\xi$ may be inferred from Eqs. (51). Thus, measurements of noise correlations can lead to information about the optical forces responsible for the longitudinal optical binding.

Different types of dynamical effects have been studied by Gordon et al. (2008). They also studied longitudinal binding in a dual beam trap but used a generalized multipole technique to describe the light distribution outside the objects and then applied Eq. (35) to calculate the optical force on each particle. They considered hydrodynamic coupling in the form of Eq. (48) and-since they did not consider the stochastic thermal activation-their theory showed no particle oscillations if both beams were collinear. However, small lateral offset of the beams caused self-sustained oscillations of the whole optically bound cluster of particles. The frequency of these oscillations decreased with an increasing number of particles in the cluster. After removing the hydrodynamic term from the equations, the oscillations were still observed but their frequency and their damping rate decreased.

\section{FURTHER EXPERIMENTAL STUDIES AND CONFIGURATIONS}

In a series of papers, Metzger et al. explored the dynamics and interactions between two and six microparticles held in a dual beam fiber trap (Metzger, Dholakia, and Wright, 2006; Metzger, Wright, and Dholakia, 2006; Metzger, Wright, Sibbett, and Dholakia, 2006; Metzger et al., 2007). Careful investigation of the equilibrium positions in this system revealed a hitherto unsuspected complexity: namely, they observed bistability in the intersphere separations dependent on the refractive index difference between the spheres and the host medium, and hysteresis in the particle equilibrium separations as the fiber separation was varied adiabatically (Metzger, Dholakia, and Wright, 2006). These observations matched well with numerical simulations based on the coupled equations for the light propagation [Eq. (41)] and the forces acting on the spheres based on Eq. (37) (Metzger, Wright, and Dholakia, 2006). Bistability and bifurcation are ubiquitous in many physical and biological systems and they are closely linked with the concept of feedback. Competition between parameters such as dispersion and nonlinearity in a wide variety of physical systems can ultimately lead to the coexistence of several stable solutions that may each be energetically favorable.

In the optical domain bistability is usually linked with the notion of nonlinearity but one can also observe classical bistability with no explicit nonlinearity, for example, the radiation pressure from the intracavity field on a moving mirror. The observations of Metzger et al. for a two-particle optically bound system constitute the first realization of bifurcation and bistability that is inherently linked with the coupled nature of the problem and the direct interplay between radiation pressure and the light redistribution by each constituent microsphere with accompanying positive feedback.

The light redistribution is a major feature of optical binding: however, in virtually all light-matter interaction experiments it is difficult if not impossible to visualize the light itself. Naturally for optically bound matter this would be useful given the importance of the light redistribution in the various phenomena observed. Metzger and colleagues (Metzger, Wright, Sibbett, and Dholakia, 2006) addressed this issue using a dye dissolved in the liquid medium and an ultrashort pulsed femtosecond laser to perform binding studies (Dholakia et al., 2004). The use of the femtosecond laser permitted two-photon excitation of the fluorescein dye within the liquid. Given the fact that the two-photon effect is strongly intensity dependent the observed fluorescence gave good signature of the light intensity distribution, decoupled from the excitation wavelength. This in turn permitted one to visualize the particle spacing as well as the actual light redistribution in the chamber. 

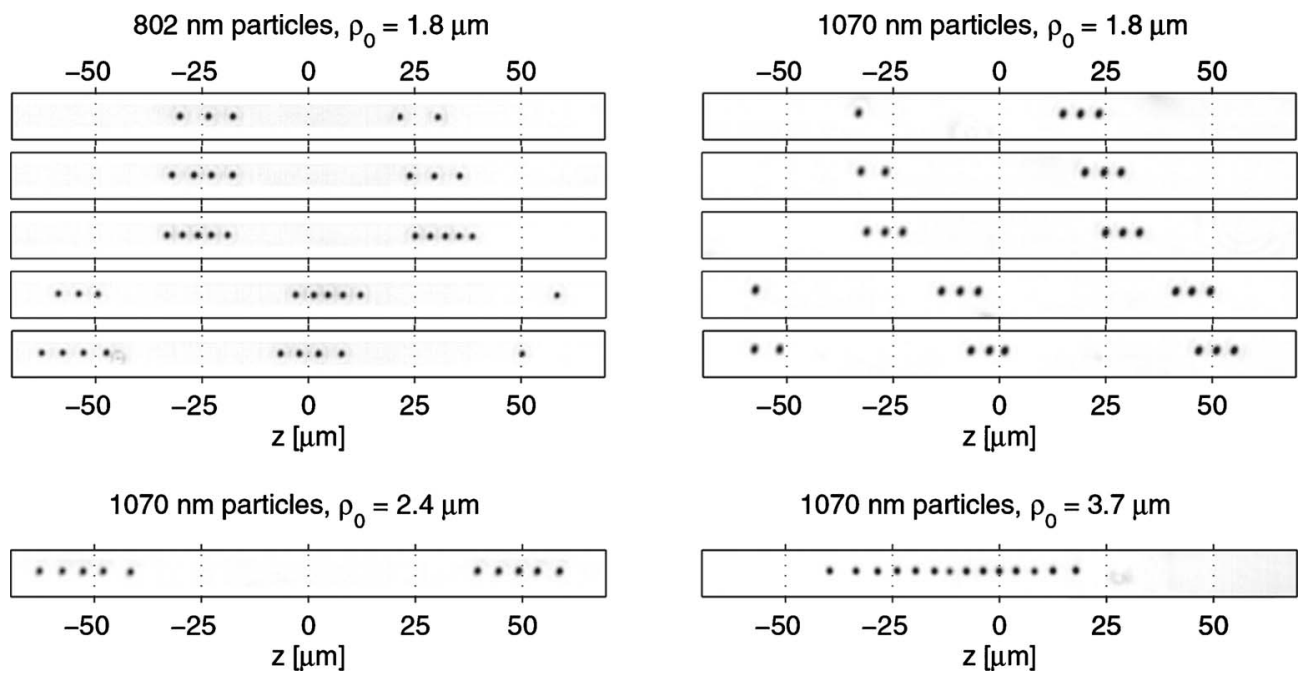

FIG. 12. Short- and long-range particle organization in counterpropagating Bessel beams. Top: Formation of chains with multiple particles of diameter $802 \mathrm{~nm}$ (left) or $1070 \mathrm{~nm}$ (right). Bottom: Self-organization of $1070 \mathrm{~nm}$ particles in wider Bessel beam cores of radii $\rho_{0}=2.4 \mu \mathrm{m}$ (left) and $\rho_{0}=3.7 \mu \mathrm{m}$ (right). From Karásek et al., 2008.

As described optical binding has been extended over longer interaction distances using light immune to diffraction, namely, the Bessel light beams. In one study (Garcés-Chávez et al., 2004), a vertically oriented zerothorder Bessel light beam created a one-dimensional array of trapped colloidal objects over extended (millimeterrange) distances. The particles were observed to align with the beam and have equilibrium positions over the entire length of the Bessel beam. This is a consequence of the interplay between optical scattering and the selfhealing properties of the Bessel beam. The study can be considered as an example of optically bound matter that is created solely by the radiation pressure and gradient forces of one light beam and its interplay with gravity. Later studies by Karásek et al. (2008) looked at two counterpropagating Bessel modes and the creation of short and long particle chains (see Fig. 12).

Separate studies by Gherardi et al. (2008) explored the use of photonic crystal fibers to create novel forms of dual beam traps where the properties of the fiber were exploited. The fiber tends itself to being "endlessly" single mode over a large range of wavelengths and thus one could create bound matter at a number of wavelengths. Interestingly one could launch multiple wavelengths simultaneously into the fiber with the use of a "white light" laser (supercontinuum beam). The low temporal coherence length of the field which was smaller than the typical particle separation caused the interparticle optical cross-talk mediated by the backscattered light to be obviated. The overall observed behavior is reminiscent of the studies carried out in monochromatic longitudinal optical binding configuration using two counterpropagating Bessel beams (Karásek et al., 2008). In particular, the chains with larger numbers of particles are more closely packed and the interparticle separations in the center of the chain are smaller than on the chain periphery. This interference exists when using a coherent source results in multistability in the in- terparticle separations with a period of the standing wave. The coherence length of the supercontinuum source is less than the interparticle separation, leading to interference suppression. From the experimental analysis no evidence of such multistability for parameter choices was seen.

\section{A. Surface (evanescent field) optical binding}

When a light beam passes through an interface from an optically dense media to one which is optically lessdense (of lower refractive index) media, it is totally reflected when its angle of incidence is above the critical angle for what is termed total internal reflection. Although in this case no wave per se propagates into the less-dense medium, there is an electromagnetic field called an evanescent wave present in the less-dense medium that decays exponentially away from the surface, with a decay length on the order of the optical wavelength. One powerful and versatile experimental geometry for multiple-particle optical binding has been the use of surface traps where the light is delivered to the particles in the form of an evanescent wave. In general evanescent wave geometries have become an important topic within the last five years. The Kretschmann geometries, the use of total internal reflection microscope objectives, and the appropriate waveguides have all offered a range of methods for generating evanescent field traps. In 1992, Kawata and Sugiura demonstrated that the evanescent field of an infrared laser could attract particles toward the surface and subsequently guide them along the surface (Kawata and Sugiura, 1992). Such behavior arises as a single laser beam at oblique incidence upon the surface has a component of momentum parallel to the surface that imparts a lateral force on a microscopic particle (akin to a radiation pressure) and drives the particles along the surface. In turn the particle 
converts the evanescent component of the light field into a traveling wave component.

In this geometry a stable evanescent wave trap can be generated using two counterpropagating laser beams so that the tangential radiation pressure on the particles averages to zero and the gradient force acting toward the surface retains the particles in the overlap region between both beams. However, the first evanescent field optical trap was realized with a total internal reflection microscope objective ( $\mathrm{Gu}$ et al., 2004). Subsequently Garcés-Chávez et al. extended Kawata's work using two beams and generating a large area trap (Garcés-Chávez et al., 2005). They also patterned the surface with a Ronchi ruling and, as expected, observed the particles $(5 \mu \mathrm{m}$ in diameter, five times the laser wavelength) to line up with the projected light fringes.

Counterpropagating evanescent waves were used for optical binding studies by Šerý et al. (2005) and Šiler et al. (2006). In their case, the illuminated area was about $40 \mu \mathrm{m}$ long and $10 \mu \mathrm{m}$ wide and they observed the formation of chains of many particles with correlated lateral motion. Smaller particles (with diameter of $350 \mathrm{~nm}$ ) were very close to each other and their interparticle distances were difficult to see; however, larger particles of diameters $520 \mathrm{~nm}, 701 \mathrm{~nm}$, and $802 \mathrm{~nm}$ settled with wellresolved distances. A subsequent key experiment in this geometry carried out by Mellor and Bain (2006) and Mellor et al. (2006) with wider beams led to the observation of radically different and counterintuitive behavior. In contrast to the previous observations, they demonstrated that small microscopic particles in such counterpropagating beams can self-assemble into ordered 2D arrays with either a rectangular or pseudohexagonal lattice. This series of experiments illustrates the sensitivity of optical binding to a multitude of parameters and shows how, by a variation in one or two parameters in an equivalent optical system, radically different experimental outcomes can be achieved.

We now examine the Mellor and Bain experiments in more detail. In the first set of experiments they projected a set of linear interference fringes upon the surface of a prism. As expected $500 \mathrm{~nm}$ diameter spheres suspended in water were seen to migrate toward the region of the linear fringes. Initially the spheres performed Brownian motion along any given fringe but as the particle density increased, the particles started lining up orthogonally to the fringe direction. Indeed as more spheres migrated to the linear fringe pattern it started turning into a saw-tooth-like arrangement and ultimately a chessboard-type pattern. They inferred that optical binding was dominating the organization of particles for their geometry. They linked their studies to those of Garcés-Chávez et al. by exploring the range of particle sizes and the typical behavior seen. When they used particles of diameter $700 \mathrm{~nm}$ or greater they saw simple alignment along the fringes as observed by Garcés-Chávez et al., however, when they used smaller objects (500 nm diameter or less) the lines evolved into saw-tooth-like patterns resulting in an overall checkerboardlike configuration. A surprising key observation was that the particle organization through the optical binding mechanism could be induced without any pattering of the light field incident upon the substrate. In particular 460 and $520 \mathrm{~nm}$ particles formed pseudohexagonal arrays rather than rectangular unit cells, where the packing fraction was $6 \%$ higher than the rectangular cell. Other particle sizes $(390 \mathrm{~nm})$ formed square arrays (regardless of the patterning upon the evanescent wave surface). They observed experimentally that the formation of optically bound matter was sensitive to the effective particle size which could be tuned by altering the ionic strength of the solution: by increasing the ionic strength the electrostatic particle repulsion is screened and the particle separation decreases. They pointed toward possible schemes for modeling the observed behavior based largely on the Mie-Debye theory which is appropriate for particles that are approximately the size of the illuminating wavelength. The challenge here is to determine a self-consistent solution for a large number of Mie scatterers placed in such an electromagnetic field. New work by this team has already started and shed some light on this process from a theoretical point of view (Taylor et al., 2008).

Mellor and colleagues extended this work in a detailed experimental study where the role of the light polarization on the bound array formation was explored (Mellor et al., 2006). Particle sizes from 390 to $520 \mathrm{~nm}$ were explored and a variety of cell geometries including rectangular arrays, three forms of hexagonal arrays, and a defective array with one row missing was observed. These studies showed that optical binding can indeed dominate over conventional optical trapping with startling consequences: it can lead to the formation of particle arrays at odds with the geometry of the illuminating interference fringes. The observed array symmetries as well as the lattice spacings within the arrays were dependent on both light field polarization and particle size. By rotating the polarization by $90^{\circ}$, "phase transitions" between different array geometries were observed (see Fig. 13).

Optical fields used for the optical trapping and binding experiments can be significantly enhanced through the exploitation of free electron oscillations in a metallic structure. Such surface plasmon polaritons (SPPs) allow the use of combined optical and thermal forces for largescale ordering of colloidal aggregations. Using an SPPbased experimental setup, Garcés-Chávez et al. (2006) explored three different regimes that led to the colloidal aggregation. The observed outcome was governed by judicious choice of the sample chamber thickness and incident SPP excitation optical power (see Fig. 14). In particular if thermal effects (convection) were suppressed through the use of chambers of thin depth, particles were seen to accumulate at the center of the excitation region and arrange into linear colloidal chains, attributed to a bindinglike behavior much like in the studies of Reece et al. (2007) which will be described next.

The results presented demonstrate that evanescent wave arrangement enables creation of optically bound structures similar to photonic crystals. However, in clas- 


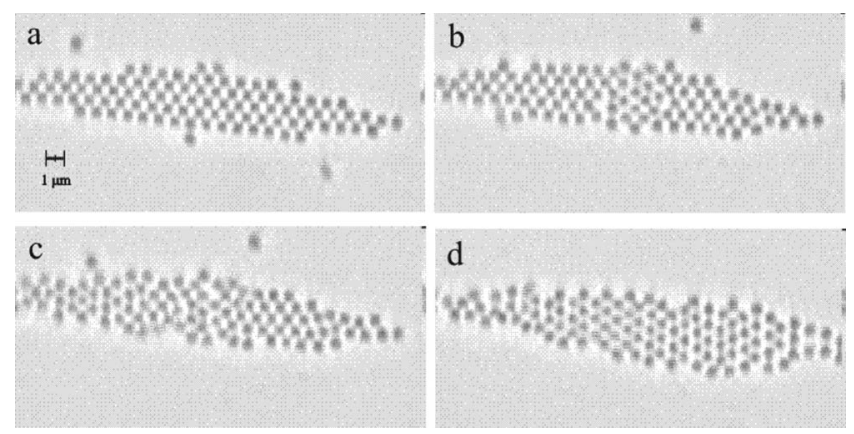

FIG. 13. Particle self-organization due to optical binding in the vicinity of a planar interface. Sequence of video frames of an array of $520 \mathrm{~nm}$ particles as a quarter-wave plate is rotated to change the polarization of one of the counterpropagating beams from $s\left(\phi=0^{\circ}\right)$ to $p\left(\phi=45^{\circ}\right)$. Hexagonal packing nucleating at the center of the array is seen at (b) and then the hexagonal crystalline structure grows outward toward the leftand right-hand sides of the array. From Mellor et al., 2006.

sical photonic structures the refractive index inhomogeneities are fixed and cannot be tuned. In contrast optically bound photonic crystals are under the influence of the forming or propagating light and can change their shape and consequently optical properties. This application is in its infancy and just a few theoretical studies are available (Povinelli, Johnson, et al., 2005; Povinelli, Loncar, et al., 2005).

\section{B. Optical binding and nonlinearity}

It is interesting that complex phenomenon such as the optical interaction among many particles in a liquid medium was observed by Ashkin et al. in their first experiments. This realization led to various experiments with colloidal suspensions that included four-wave mixing (Smith et al., 1981), self-focusing optical beams (Ashkin et al., 1982), optical spatial soliton (OSS) propagation (Yashin et al., 2005), and modulation instability (MI) (Reece et al., 2007). How can we interpret the origin of this nonlinearity? In the presence of a continuous-wave optical field with inhomogeneous intensity profile $I(r)$ dielectric particles are exposed to the optical gradient (dipole) force $F_{\text {grad }}=\nabla_{r} U(r)$, with $U(r)=\alpha^{\prime} I(r) / 4$ denoting the dipole interaction energy, $\alpha^{\prime}$ being the real part of the particle polarizability in the liquid given by Eq. (14). For the typical situation of microparticles and nanoparticles of refractive index higher than the surrounding liquid, $\alpha^{\prime}>0$, the gradient force attracts the particles toward the regions of high optical intensity. The optical nonlinearity of dielectric nanoparticle suspensions then arises from the intensity-dependent modulation of the local nanoparticle density and, consequently, the local refractive index of the particle suspension. Thus one can consider colloidal suspensions as the artificial Kerr media in which the induced change in refractive index is proportional to the applied light intensity: $\Delta n(r)=n_{2 K} I(r)$, with $n_{2 K}$ the nonlinear Kerr coefficient.

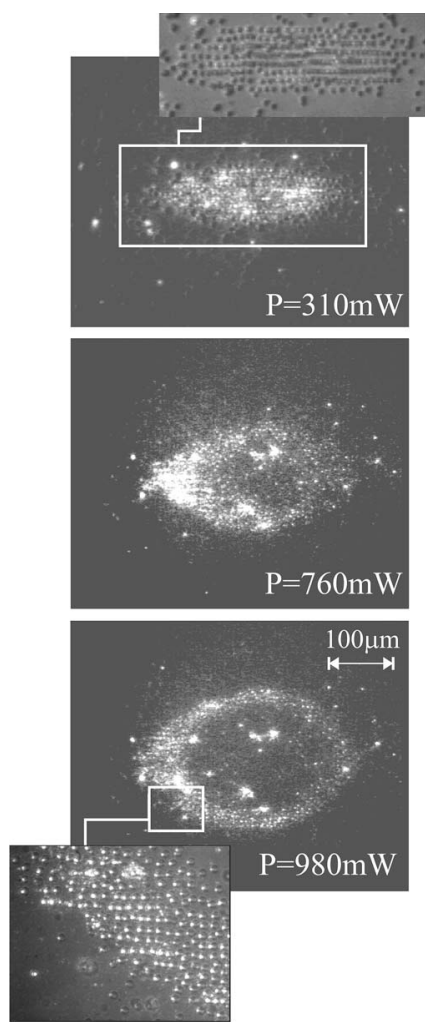

FIG. 14. Particle self-organization due to the combination of optical binding and thermophoresis in the vicinity of a metalcoated planar interface. Increasing the incident light power in a thin chamber (with height of $10 \mu \mathrm{m}$ ) formed above a metalcoated interface triggers lateral thermophoresis that results in the repulsion of particles (silica, with diameter of $5 \mu \mathrm{m}$ ) from the center of the illuminated region where the SPPs are excited (hot to cold movement). Subsequently the repelled colloids arrange into linear arrays at the periphery of the region. Light scattered by the colloids (no additional white light illumination). The top and bottom insets are detailed views of the linear ordering of the accumulated particles acquired with additional white light illumination. From Garcés-Chávez et al., 2006.

Despite the fact the artificial Kerr media model has been successfully applied to experiments on nonlinear optics in colloidal suspensions, the nature of the nonlinearity for this soft condensed matter system is still under much debate (Conti et al., 2005; El-Ganainy, Christodoulides, Musslimani, et al., 2007; El-Ganainy, Christodoulides, Rotschild, and Segev, 2007; Gordon et al., 2007; Matuszewski et al., 2008, 2009). The crucial point is that the electrostriction model for the nonlinear optical response ultimately leads to an exponential model for the change of a colloidal suspension refractive index at temperature $T$ (Smith et al., 1982),

$$
\Delta n(I)=\Delta n(I=0) \exp \left[\frac{\alpha^{\prime} I}{4 k_{B} T}\right] .
$$

The Taylor expansion for small argument of Eq. (53) gives the coefficient of the Kerr nonlinear optical response proportional to the optical intensity $I$ of the beam but of course it is part of an infinite expansion of 
higher-order nonlinearities. Is such an exponential model an accurate representation of the nonlinearity of colloidal suspensions? How might we access or demonstrate the higher-order nonlinearities in an experimental scenario? More broadly how does this link affect the topic of optical binding? The resolution of these questions is not immediately evident: notably the exponential model is a single-particle model that neglects particle-particle interactions (optical binding) which in turn can limit the compressibility of the liquid suspension, potentially limiting the nonlinear optical response of the soft condensed matter system to the leading Kerr term. For four-wave mixing experiments this Kerr approximation is acceptable if the incident optical intensities are controlled such that $n_{2 K} I \ll 1$. Recent studies of Conti et al. (2005) introduced a nonlocal Kerr nonlinear model for generic soft condensed matter systems that yields stable OSSs. However, they did not address how such an approach may link to the exponential model for colloidal suspensions. Further studies by El-Ganainy, Christodoulides, Musslimani, et al. (2007) and ElGanainy, Christodoulides, Rotschild, and Segev (2007) and independently by Gordon et al. (2007) suggested that the full exponential model should be employed to investigate OSS in colloidal suspensions. The caveat in this approach is that it will only apply to low colloidal densities, although how one interprets the definition of "low" is somewhat unclear. A crucial point is that including the full exponential model renders the OSS highly unstable due to the supercritical self-focusing collapse that arises for higher-order self-focusing nonlinearities (Kosmatov et al., 1991). This contradicts recent experimental observations of relatively stable OSS that seem to agree reasonably with the Kerr model (Yashin et al., 2005; Reece et al., 2007). Separately, Matuszewski et al. $(2008,2009)$ treated theoretically the colloidal suspension as a hard sphere gas. This permitted them to incorporate the compressibility of the system. The net result of such an approach is the prediction of saturating the exponential nonlinearity at high intensities.

Recent theoretical and experimental studies (Hansen et al., 2005; Reece et al., 2007; Lee et al., 2009) indicated that the exponential nonlinearity model seems to have the lowest correlation with experiments, followed by the artificial Kerr medium approach (Lee et al., 2009). Theoretical nonideal gas based model including the effects of the limited compressibility of the particle suspension agreed well with the experimental results. While this is an important step forward it is still unclear how the granularity of the colloidal suspensions enters the nonlinear optical response and how the theoretical approaches in optical binding might be reconciled with such studies. When the laser beam waist and mean particle-particle distances are comparable, one might need to invoke the nonlocal nonlinear model of Conti et al. (2005) that could lead to a further softening of the nonlinear optical response in comparison with the exponential model.

Reece et al. $(2006,2007)$ added another twist to the problem. In 2007 they explored the behavior of $410 \mathrm{~nm}$

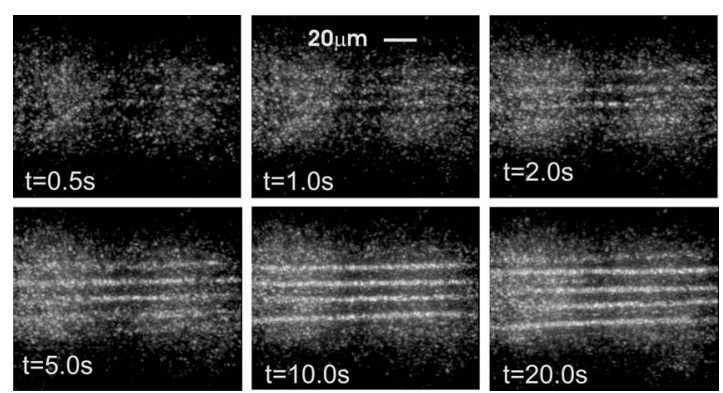

FIG. 15. Formation of OSSs under unpatterned plane-wave illumination. The temporal evolution of the OSS created from polymer particles of diameter $410 \mathrm{~nm}$ formation for illuminating powers above the threshold. Each panel is a CCD image of the illuminated region at different times after the light has been coupled into the system. Bright-field imaging also confirms the presence of ordered colloids in regions of high intensity. From Reece et al., 2007.

diameter particles in a surface geometry with unpatterned illumination based on two counterpropagating beams that balanced their radiation pressures and prevented net motion of particles along the surface away from the highest intensity regions. For the experimental work a prism-coupled resonant dielectric waveguide was used to generate counterpropagating $(\mathrm{CP})$ waveguide modes with an evanescent wave (EW) component extending into the colloidal suspension (see Fig. 15). Transverse optical gradient forces due to the EW acting on particles in the proximity of the supporting surface resulted in the accumulation of particles at the center of the illuminated area, where the scattering forces due to the CP fields along the propagation $x$ axis were balanced. In contrast to the work of Mellor et al. they observed particles organizing into parallel linear arrays in the absence of any illuminating light intensity patterning. Interestingly they attributed this trend to a nonlinear interparticle interaction and interpreted the results as the observation of modulation instability and the onset of solitonlike behavior in the colloidal sample.

Optical spatial solitons are spatially localized, nondiffracting light propagation modes that may exist in nonlinear optical media. They occur as a result of the balance between diffraction and nonlinearity connected with the refractive index increase in the high-intensity parts of the optical field that results in self-focusing under intense illumination (Ashkin et al., 1982). For a plane-wave incident field, small wave front perturbations can cause the optical field to break up into periodic arrays of OSS or more complex patterns, an effect known as MI. Both OSS and MI are generic properties of the wave propagation governed by the nonlinear Schrödinger equation. In addition to nonlinear optics, there are numerous examples in other physical systems where such solitary waves and related phenomena are observed particularly in pattern formation in granular systems and complex fluids.

An interesting parallel can be drawn between the OSS and MI interpretation of optical force induced selforganization of colloidal dispersions and optical force 
mediated particle-particle interactions observed in optical binding, particularly in light of the recent reports of two-dimensional checkerboardlike array formation in a trapping geometry similar to the one described above. An interpretation of the behavior of optically bound colloidal systems from a nonlinear systems approach may provide further insights into the observed pattern formation for such lateral binding. MI in nonlinear systems may also lead to more complex pattern formation, such as two-dimensional square lattices and hexagonal and spiral type formations; this may be achieved by driving the system well above the threshold.

\section{Experimental studies of optical binding of nanoparticles}

Previous results have indicated that optical binding between nanoparticles is highly dependent on used geometries and shows behavior dictated by polarization and wavelength of the illuminating light. Naturally Brownian motion must be overcome for particles to attain stable equilibrium positions (Zelenina et al., 2007).

In the experiments of Svedberg et al. (2006) metallic nanoparticle pairs were created to study surface enhanced Raman spectroscopy. They explored the behavior of the system in which one trapped nanoparticle was brought in close vicinity of another adhered nanoparticle. The immobilized particle affected the potential energy landscape of the system and a second deep potential well for the trapped particle confinement was seen that was attributed to optical binding. This meant the trapped particle might "hop" between both potential wells. Electrodynamic calculations backed up this preliminary observation.

In later and related work, Dienerowitz et al. explored the behavior of two nanoparticles inside a dark trap created by an annular Laguerre-Gaussian beam (Dienerowitz et al., 2008). In this instance the particles were confined via scattering of the light field of the beam that was tuned below the plasmon resonance of the particles. The observed particles did not attract each other or amalgamate as typically observed in traditional optical tweezers; rather repulsion between the particles was observed with particles assuming opposite positions $\left(180^{\circ}\right.$ apart) in the trap. Due to the use of the LaguerreGausian beam particle orbiting was also observed. They attributed this behavior to an optical binding type interaction between the objects. A similar configuration has been studied by Bradshaw and Andrews (2005b) using QED calculations. Further work here may show more clearly links between experiment and theory.

\section{Optical binding in air}

While the vast majority of experiments in optical micromanipulation are performed in a liquid medium, trapping is also possible in air and vacuum. The original studies were conducted in the 1970s again by Ashkin and Dziedzic (1975, 1976) and Ashkin (2006). Trapping in air may lead to the trapped particle acting as an underdamped harmonic oscillator and show behavior markedly different from the typical overdamped case. Interesting experiments have been performed recently in the area of optical binding in gaseous environment. Guillon et al. reported the generation of arrays of microdroplets in air (Guillon et al., 2006). Their work concentrated on the trapping of small droplets in a counterpropagating laser beam geometry where the trapping occurs in the region with near equal irradiance from both beams. The strong refractive index contrast distinguished these studies from optical binding in liquid medium. This first study and analysis proved somewhat contentious (Guillon et al., 2008; Liu and Yu, 2008) and an erratum (Guillon et al., 2007) in fact stated that there was some misinterpretation of the data: the doublets seen were in fact single particles and the three particles were in fact only two. However, the spectroscopic studies of the scattering resonances fitted well the Mie scattering theory and allowed an accurate determination of the droplet radii. Subsequent studies by Guillon sought to make detailed measurements of this phenomenon. These studies looked at both direct and spectroscopic imaging of optically trapped Mie droplets. Light scattering by the droplets led to "glare" points around the droplet's azimuth. They explored oil and salt water droplets trapped in counterpropagating geometries with $\approx 150 \mathrm{~mW}$ at $532 \mathrm{~nm}$. Orthogonal $\left(90^{\circ}\right)$ elastic light scattering from the droplets gave images that were fitted with theory. The droplet radius was determined by fitting whispering gallery mode resonances to the theoretical Mie model. The observed glare points were dependent on the angular momentum number of the excited resonance mode.

\section{CONCLUSIONS}

The light-matter interaction has been at the core of exceptional scientific advances and insights in the past 40 years. The broad topic of optical micromanipulation has impacted the fields of colloidal science, biomedical research, atom optics, and quantum gases. For particles with sizes from the nanoscale upward, the traditional and well-known gradient and scattering forces have recently been supplemented with optical binding: essentially an optically mediated particle-particle interaction. Although still at its infancy, this research area has already seen some fascinating and complex particle behavior. Optical binding has brought further complexity to the realm of relevant forces governing interactions between colloidal particles and it can contribute significantly to the spontaneous organization of the colloids that serve as increasingly popular model systems for the solid-state phenomena. A number of numerical approaches have been developed to understand this experimentally driven topic and some good agreement between the observations and theory has already been observed. In this area the paraxial theory and CDM approaches have gained prominence and applicability but have limitations and it would be important to try and design and implement experiments that can look at the detailed predictions of more exact theoretical ap- 
proaches. Intriguingly the light-particle interaction is "nonlinear" due to inherent feedback and leads to interesting multistable configurations as well as behavior reminiscent of nonlinear media, namely, modulation instability and the emergence of optical solitons. Despite the recent progress much work still needs to be done to unify the various mathematical approaches and truly engineer and utilize the binding interaction to create templates and arrays in controlled configurations at will. This remains a major task and much work needs to be accomplished but the basic framework is certainly in place.

\section{ACKNOWLEDGMENTS}

The authors gratefully acknowledge the input and comments of Dr. Alexandr Jonáš and Professor Ewan Wright and the support from CSF (Grant No. GA202/ 09/0348), MEYS CR (Grant No. LC06007), ISI IRP (Grant No. AV0Z20650511), and the U.K. Engineering and Physical Sciences Research Council. K.D. is a Royal Society-Wolfson Merit Award holder.

\section{REFERENCES}

Akselrod, G. M., W. Timp, U. Mirsaidov, Q. Zhao, C. Li, R. Timp, K. Timp, P. Matsudaira, and G. Timp, 2006, Biophys. J. 91, 3465.

Andrews, D. L., and D. S. Bradshaw, 2005, Opt. Lett. 30, 783.

Andrews, D. L., R. G. Crisp, and D. S. Bradshaw, 2006, J. Phys. B 39, S637.

Arias-González, J. R., and M. Nieto-Vesperinas, 2003, J. Opt. Soc. Am. A 20, 1201.

Ashkin, A., 1970, Phys. Rev. Lett. 24, 156.

Ashkin, A., 1992, Biophys. J. 61, 569.

Ashkin, A., 2006, Optical Trapping and Manipulation of Neutral Particles Using Lasers (World Scientific, London).

Ashkin, A., and J. M. Dziedzic, 1975, Science 187, 1073.

Ashkin, A., and J. M. Dziedzic, 1976, Appl. Phys. Lett. 28, 333.

Ashkin, A., J. M. Dziedzic, J. E. Bjorkholm, and S. Chu, 1986, Opt. Lett. 11, 288.

Ashkin, A., J. M. Dziedzic, and P. W. Smith, 1982, Opt. Lett. 7, 276.

Bartlett, P., S. I. Henderson, and S. J. Mitchell, 2001, Philos. Trans. R. Soc. London 359, 883.

Barton, J. P., D. R. Alexander, and S. A. Schaub, 1989, J. Appl. Phys. 66, 4594.

Benito, D. C., S. H. Simpson, and S. Hanna, 2008, Opt. Express 16, 2942.

Born, M., and E. Wolf, 1999, Principles of Optics (Pergamon, London).

Bradshaw, D. S., and D. L. Andrews, 2005a, Opt. Lett. 30, 3039.

Bradshaw, D. S., and D. L. Andrews, 2005b, Phys. Rev. A 72, 033816.

Bradshaw, D. S., and D. L. Andrews, 2006, Phys. Rev. A 73, 039903.

Buican, T. N., M. J. Smyth, H. A. Crissman, G. C. Salzman, C. C. Stewart, and J. Martin, 1987, Appl. Opt. 26, 5311.

Burns, M. M., J.-M. Fournier, and J. A. Golovchenko, 1989, Phys. Rev. Lett. 63, 1233.

Burns, M. M., J.-M. Fournier, and J. A. Golovchenko, 1990,
Science 249, 749.

Chaumet, P. C., and C. Billaudeau, 2007, J. Appl. Phys. 101, 023106.

Chaumet, P. C., and M. Nieto-Vesperinas, 2000a, Opt. Lett. 25, 1065.

Chaumet, P. C., and M. Nieto-Vesperinas, 2000b, Phys. Rev. B 61, 14119.

Chaumet, P. C., and M. Nieto-Vesperinas, 2000c, Phys. Rev. B 62, 11185.

Chaumet, P. C., and M. Nieto-Vesperinas, 2001, Phys. Rev. B 64, 035422 .

Chaumet, P. C., A. Sentenac, and A. Rahmani, 2004, Phys. Rev. E 70, 036606.

Chu, S., 1998, Rev. Mod. Phys. 70, 685.

Cohen-Tannoudji, C. N., 1998, Rev. Mod. Phys. 70, 707.

Collett, W. L., C. A. Ventrice, and S. M. Mahajan, 2003, Appl.

Phys. Lett. 82, 2730.

Constable, A., and J. Kim, 1993, Opt. Lett. 18, 1867.

Conti, C., G. Ruocco, and S. Trillo, 2005, Phys. Rev. Lett. 95, 183902.

de Groot, S. R., and L. G. Suttorp, 1971, Foundations of Electrodynamics (North-Holland, Amsterdam).

Depasse, F., and J.-M. Vigoureux, 1994, J. Phys. D 27, 914.

Derjaguin, B., and L. Landau, 1941, Acta Physicochim. URSS 14, 633.

Dholakia, K., and W. M. Lee, 2008, Adv. At. Mol. Phys. 56, 261.

Dholakia, K., H. Little, C. T. A. Brown, B. Agate, D. McGloin, L. Paterson, and W. Sibbett, 2004, New J. Phys. 6, 136.

Dienerowitz, M., M. Mazilu, P. J. Reece, T. F. Krauss, and K. Dholakia, 2008, Opt. Express 16, 4991.

Draine, B., 1988, Astrophys. J. 333, 848.

Draine, B., and J. C. Weingartner, 1996, Astrophys. J. 470, 551. Draine, B. T., and P. J. Flatau, 1994, J. Opt. Soc. Am. A Opt. Image Sci. Vis 11, 1491.

Durnin, J., J. J. Miceli, and J. H. Eberly, 1987, Phys. Rev. Lett. 58, 1499.

El-Ganainy, R., D. N. Christodoulides, Z. H. Musslimani, C. Rotschild, and M. Segev, 2007, Opt. Lett. 32, 3185.

El-Ganainy, R., D. N. Christodoulides, C. Rotschild, and M. Segev, 2007, Opt. Express 15, 10207.

Foldy, L. L., 1945, Phys. Rev. 67, 107.

Fujiwara, H., H. Takasaki, J.-I. Hotta, and K. Sasaki, 2004, Appl. Phys. Lett. 84, 13.

Garcés-Chávez, V., K. Dholakia, and G. C. Spalding, 2005, Appl. Phys. Lett. 86, 031106.

Garcés-Chávez, V., R. Quidant, P. J. Reece, G. Badenes, L. Torner, and K. Dholakia, 2006, Phys. Rev. B 73, 085417.

Garcés-Chávez, V., D. Roskey, M. D. Summers, H. Melville, D. McGloin, E. M. Wright, and K. Dholakia, 2004, Appl. Phys. Lett. 85, 4001.

Garcia de Abajo, F. J. G., 2007, Rev. Mod. Phys. 79, 1267.

Gaugiran, S., S. Gétin, J. M. Fedeli, G. Colas, A. Fuchs, F. Chatelain, and J. Dérouard, 2005, Opt. Express 13, 6956.

Gaugiran, S., S. Getin, J. M. Fedeli, and J. Derouard, 2007, Opt. Express 15, 8146.

Gauthier, R. C., 2005, Opt. Express 13, 3707.

Gherardi, D. M., A. E. Carruthers, T. Čižmár, E. M. Wright, and K. Dholakia, 2008, Appl. Phys. Lett. 93, 041110.

Goodman, J. J., B. T. Draine, and P. J. Flatau, 1991, Opt. Lett. 16, 1198.

Gordon, R., J. T. Blakely, and D. Sinton, 2007, Phys. Rev. A 75, 055801. 
Gordon, R., M. Kawano, J. T. Blakely, and D. Sinton, 2008, Phys. Rev. B 77, 245125.

Grzegorczyk, T. M., B. A. Kemp, and J. A. Kong, 2006a, Opt. Lett. 31, 3378.

Grzegorczyk, T. M., B. A. Kemp, and J. A. Kong, 2006b, Phys. Rev. Lett. 96, 113903.

Grzegorczyk, T. M., B. A. Kemp, and J. A. Kong, 2006c, J. Opt. Soc. Am. A Opt. Image Sci. Vis 23, 2324.

Gu, M., J.-B. Haumonte, Y. Micheau, J. W. M. Chon, and X. Gan, 2004, Appl. Phys. Lett. 84, 4236.

Gu, M., P. Ke, and X. Gan, 1997, Rev. Sci. Instrum. 68, 3666. Guillon, M., 2006, Opt. Express 14, 3045.

Guillon, M., O. Moine, and B. Stout, 2006, Phys. Rev. Lett. 96, 143902.

Guillon, M., O. Moine, and B. Stout, 2007, Phys. Rev. Lett. 99, 079901.

Guillon, M., B. Stout, and O. Moine, 2008, Phys. Rev. Lett. 100, 199404.

Gussgard, R., T. Lindmo, and I. Brevik, 1992, J. Opt. Soc. Am. B 9, 1922.

Hansen, P. M., V. K. Bhatia, N. Harrit, and L. Oddershede, 2005, Nano Lett. 5, 1937.

Harada, Y., and T. Asakura, 1996, Opt. Commun. 124, 529.

Hoekstra, A. G., M. Frijlink, L. B. F. M. Waters, and P. M. A. Sloot, 2001, J. Opt. Soc. Am. A Opt. Image Sci. Vis 18, 1944. Jákl, P., M. Šerý, J. Ježek, A. Jonáš, M. Liška, and P. Zemánek, 2003, J. Mod. Opt. 50, 1615.

Jonáš, A., and P. Zemánek, 2008, Electrophoresis 29, 4813.

Jonáš, A., P. Zemánek, and E.-L. Florin, 2001, Opt. Lett. 26, 1466.

Karásek, V., O. Brzobohatý, and P. Zemánek, 2009, J. Opt. A, Pure Appl. Opt. 11, 034009.

Karásek, V., T. Cižmár, O. Brzobohatý, P. Zemánek, V. GarcésChávez, and K. Dholakia, 2008, Phys. Rev. Lett. 101, 143601.

Karásek, V., T. Cižmár, and P. Zemánek, 2006, in Nanophotonics, edited by J.-M. N. D. L. Andrews and A. Ostendorf, Proceedings of SPIE Vol. 6195 (SPIE, Bellingham, WA), pp. 632-609.

Karásek, V., K. Dholakia, and P. Zemánek, 2006, Appl. Phys. B: Lasers Opt. 84, 149.

Karásek, V., and P. Zemánek, 2007, J. Opt. A, Pure Appl. Opt. 9, S215.

Kawata, S., and T. Sugiura, 1992, Opt. Lett. 17, 772.

Kemp, B., T. Grzegorczyk, and J. Kong, 2005, Opt. Express 13, 9280.

Kemp, B. A., T. M. Grzegorczyk, and J. A. Kong, 2006, Phys. Rev. Lett. 97, 133902.

Ketterle, W., 2002, Rev. Mod. Phys. 74, 1131.

Kosmatov, N., V. Shvets, and V. Zakharov, 1991, Physica D 52, 16.

Lax, M., 1951, Rev. Mod. Phys. 23, 287.

Lax, M., 1952, Phys. Rev. 85, 621.

Lee, W. M., R. El-Ganainy, D. N. Christodoulides, K. Dholakia, and E. M. Wright, 2009, Opt. Express 17, 10277.

Liu, S., and J.-T. Yu, 2008, Phys. Rev. Lett. 100, 199403.

Mansuripur, M., 2004, Opt. Express 12, 5375.

Mansuripur, M., 2005, Opt. Express 13, 2245.

Matuszewski, M., W. Krolikowski, and Y. S. Kivshar, 2008, Opt. Express 16, 1371.

Matuszewski, M., W. Krolikowski, and Y. S. Kivshar, 2009, Phys. Rev. A 79, 023814.

Mazolli, A., P. A. M. Neto, and H. M. Nussenzveig, 2003, Proc. R. Soc. London, Ser. A 459, 3021.
McGloin, D., A. E. Carruthers, K. Dholakia, and E. M. Wright, 2004, Phys. Rev. E 69, 021403.

Meiners, J.-C., and S. R. Quake, 1999, Phys. Rev. Lett. 82, 2211.

Mellor, C. D., and C. D. Bain, 2006, ChemPhysChem 7, 329.

Mellor, C. D., T. A. Fennerty, and C. D. Bain, 2006, Opt. Express 14, 10079.

Metzger, N. K., K. Dholakia, and E. M. Wright, 2006, Phys. Rev. Lett. 96, 068102.

Metzger, N. K., P. R. T. Jess, L. Paterson, E. M. Wright, and K. Dholakia, 2005, in Optical Trapping and Optical Micromanipulation 2, edited by K. Dholakia and G. Spalding, Proceedings of SPIE Vol. 5930 (SPIE, Bellingham, WA), p. 59300M. Metzger, N. K., R. F. Marchington, M. Mazilu, R. L. Smith, K. Dholakia, and E. M. Wright, 2007, Phys. Rev. Lett. 98, 068102.

Metzger, N. K., E. M. Wright, and K. Dholakia, 2006, New J. Phys. 8, 139.

Metzger, N. K., E. M. Wright, W. Sibbett, and K. Dholakia, 2006, Opt. Express 14, 3677.

Neuman, K. C., and S. M. Block, 2004, Rev. Sci. Instrum. 75, 2787.

Neves, A. A. R., A. Fontes, C. L. Cesar, A. Camposeo, R. Cingolani, and D. Pisignano, 2007, Phys. Rev. E 76, 061917.

Ng, J., and C. T. Chan, 2006, Opt. Lett. 31, 2583.

Ng, J., C. T. Chan, P. Sheng, and Z. Lin, 2005, Opt. Lett. 30, 1956.

Ng, J., Z. F. Lin, C. T. Chan, and P. Sheng, 2005, Phys. Rev. B 72, 085130.

Nieminen, T., H. Rubinsztein-Dunlop, N. Heckenberg, and A. Bishop, 2001, Comput. Phys. Commun. 142, 468.

Nieminen, T. A., G. Knöner, N. R. Heckenberg, and H. Rubinsztein-Dunlop, 2007, Methods Cell Biol. 82, 207.

Nieminen, T. A., V. L. Y. Loke, A. B. Stilgoe, G. Knöner, A. M. Brańczyk, N. R. Heckenberg, and H. Rubinsztein-Dunlop, 2007, J. Opt. A, Pure Appl. Opt. 9, S196.

Nieto-Vesperinas, N., P. C. Chaumet, and R. Rahmani, 2004, Philos. Trans. R. Soc. London, Ser. A 362, 719.

Novotny, L., 1997a, J. Opt. Soc. Am. A Opt. Image Sci. Vis 14, 91.

Novotny, L., 1997b, J. Opt. Soc. Am. A Opt. Image Sci. Vis 14, 105.

Novotny, L., and B. Hecht, 2006, Principles of Nano-Optics (Cambridge University Press, Cambridge).

Phillips, W. D., 1998, Rev. Mod. Phys. 70, 721.

Povinelli, M., S. Johnson, M. Loncar, M. Ibanescu, E. Smythe, F. Capasso, and J. Joannopoulos, 2005, Opt. Express 13, 8286. Povinelli, M., M. Loncar, M. Ibanescu, E. Smythe, S. Johnson, F. Capasso, and J. Joannopoulos, 2005, Opt. Lett. 30, 3042.

Press, W. H., S. A. Teukolsky, W. T. Vetterling, and B. P. Flannery, 1992, Numerical Recipes in C (Cambridge University Press, Cambridge).

Purcell, E. M., and C. R. Pennypacker, 1973, Astrophys. J. 186, 705.

Reece, P. J., V. Garcés-Chávez, and K. Dholakia, 2006, Appl. Phys. Lett. 88, 221116.

Reece, P. J., E. M. Wright, and K. Dholakia, 2007, Phys. Rev. Lett. 98, 203902.

Ren, K. F., G. Gréhan, and G. Gouesbet, 1996, Appl. Opt. 35, 2702.

Rodriguez, J., and D. L. Andrews, 2009a, Opt. Commun. 282, 2267.

Rodriguez, J., and D. L. Andrews, 2009b, Phys. Rev. A 79, 
029902.

Rohrbach, A., 2005, Phys. Rev. Lett. 95, 168102.

Romero, L. C. D., J. Rodriguez, and D. L. Andrews, 2008, Opt. Commun. 281, 865.

Šerý, M., M. Šiler, T. Čižmár, P. Jákl, and P. Zemánek, 2005, in Laser and Applications, edited by K. M. Abramski, A. Lapucci, and E. P. Plinski, Proceedings of SPIE Vol. 5958 (SPIE, Bellingham, WA), p. 5958OL.

Šiler, M., T. Čižmár, M. Šerý, and P. Zemánek, 2006, Appl. Phys. B: Lasers Opt. 84, 157.

Singer, W., M. Frick, S. Bernet, and M. Ritsch-Marte, 2003, J. Opt. Soc. Am. B 20, 1568.

Smith, P., A. Ashkin, and W. Tomlinson, 1981, Opt. Lett. 6, 284.

Smith, P., P. Maloney, and A. Ashkin, 1982, Opt. Lett. 7, 347. Stratton, J. A., 1941, Electromagnetic Theory (McGraw-Hill, New York).

Svedberg, F., Z. Li, H. Xu, and M. Käll, 2006, Nano Lett. 6, 2639.

Tatarkova, S. A., A. E. Carruthers, and K. Dholakia, 2002, Phys. Rev. Lett. 89, 283901.

Taylor, J. M., L. Y. Wong, C. D. Bain, and G. D. Love, 2008,
Opt. Express 16, 6921.

Thirunamachandran, T., 1980, Mol. Phys. 40, 393.

Verwey, E., and J. Overbeek, 1948, Theory of the Stability of Lyophobic Colloids (Elsevier, Amsterdam).

Viana, N. B., M. S. Rocha, O. N. Mesquita, A. Mazolli, P. A. Maia Neto, and H. M. Nussenzveig, 2007, Phys. Rev. E 75, 021914.

White, D. A., 2000, Comput. Phys. Commun. 128, 558.

Xu, F., K. Ren, G. Gouesbet, X. Cai, and G. Gréhan, 2007, Phys. Rev. E 75, 026613.

Xu, Y.-L., 1995, Appl. Opt. 34, 4573.

Yashin, V., S. Chizhov, R. Sabirov, T. Starchikova, N. Vysotina, N. Rozanov, V. Semenov, V. Smirnov, and S. Fedorov, 2005, Opt. Spectrosc. 98, 466.

Zakharian, A. R., M. Mansuripur, and J. V. Moloney, 2005, Opt. Express 13, 2321.

Zakharian, A. R., P. Polynkin, M. Mansuripur, and J. Moloney, 2006, Opt. Express 14, 3660.

Zelenina, A. S., R. Quidant, and M. Nieto-Vesperinas, 2007, Opt. Lett. 32, 1156.

Zemánek, P., A. Jonáš, and M. Liška, 2002, J. Opt. Soc. Am. A Opt. Image Sci. Vis 19, 1025. 